\title{
Angiotensin receptors and $\beta$-catenin regulate brain endothelial integrity in malaria
}

\author{
Julio Gallego-Delgado, ${ }^{1}$ Upal Basu-Roy, ${ }^{2}$ Maureen Ty, ${ }^{1}$ Matilde Alique, ${ }^{3}$ Cristina Fernandez-Arias, ${ }^{1}$ Alexandru Movila, ${ }^{1}$ \\ Pollyanna Gomes, ${ }^{1}$ Ada Weinstock, ${ }^{4}$ Wenyue Xu, ${ }^{1}$ Innocent Edagha, ${ }^{1}$ Samuel C. Wassmer, ${ }^{1}$ Thomas Walther, ${ }^{5,6}$ \\ Marta Ruiz-Ortega, ${ }^{3}$ and Ana Rodriguez ${ }^{1}$
}

'Division of Parasitology, Department of Microbiology, New York University School of Medicine, New York, New York, USA. ²Department of Microbiology, New York University School of Medicine, New York, New York, USA. ${ }^{3}$ Cellular Biology in Renal Diseases Laboratory, Universidad Autónoma Madrid, Instituto de Investigación Fundación Jiménez Díaz, Madrid, Spain. ${ }^{4}$ Marc and Ruti Bell Program in Vascular Biology, Center for the Prevention of Cardiovascular Disease, Departments of Medicine (Cardiology) and Cell Biology, New York University School of Medicine, New York, New York, USA. ${ }^{5}$ Department of Pediatric Surgery and Department of Obstetrics, Division of Women and Child Health, Center for Perinatal Medicine, University of Leipzig, Leipzig, Germany. ${ }^{6}$ Department of Pharmacology and Therapeutics, School of Medicine and School of Pharmacy, University College Cork, Cork, Ireland.

\begin{abstract}
Cerebral malaria is characterized by cytoadhesion of Plasmodium falciparum-infected red blood cells (Pf-iRBCs) to endothelial cells in the brain, disruption of the blood-brain barrier, and cerebral microhemorrhages. No available antimalarial drugs specifically target the endothelial disruptions underlying this complication, which is responsible for the majority of malariaassociated deaths. Here, we have demonstrated that ruptured Pf-iRBCs induce activation of $\beta$-catenin, leading to disruption of inter-endothelial cell junctions in human brain microvascular endothelial cells (HBMECs). Inhibition of $\beta$-catenin-induced TCF/LEF transcription in the nucleus of HBMECs prevented the disruption of endothelial junctions, confirming that $\beta$-catenin is a key mediator of $P$. falciparum adverse effects on endothelial integrity. Blockade of the angiotensin II type 1 receptor (AT1) or stimulation of the type 2 receptor (AT2) abrogated $P f$-iRBC-induced activation of $\beta$-catenin and prevented the disruption of HBMEC monolayers. In a mouse model of cerebral malaria, modulation of angiotensin II receptors produced similar effects, leading to protection against cerebral malaria, reduced cerebral hemorrhages, and increased survival. In contrast, AT2deficient mice were more susceptible to cerebral malaria. The interrelation of the $\beta$-catenin and the angiotensin II signaling pathways opens immediate host-targeted therapeutic possibilities for cerebral malaria and other diseases in which brain endothelial integrity is compromised.
\end{abstract}

\section{Introduction}

Cerebral malaria is a multifactorial syndrome involving the interaction between Plasmodium falciparum-infected red blood cells (Pf-iRBCs) and host cerebral microvascular endothelial cells (1). P. falciparum erythrocyte membrane protein-1 (PfEMP1) expressed on the surface of iRBCs can bind to several host endothelial receptors of the cerebral microvascular bed (endothelial protein C receptor, ICAM-1; refs. 2-4), leading to their local sequestration. Disruption of the blood-brain barrier (BBB), ranging from increased permeability to complete loss of interendothelial junctions (IEJs) and to petechial hemorrhages in the brain, is a characteristic feature of cerebral malaria $(5,6)$. Available antimalarial drugs do not have specific effects against the endothelial disruption that occurs during cerebral malaria, for which adjunctive treatment is urgently needed. Our aim is to strengthen endothelial integrity to protect patients against cerebral malaria pathology while conventional antimalarial drugs eliminate the parasite.

\section{Related Commentary: p. 3725}

Conflict of interest: J. Gallego-Delgado and A. Rodriguez are the authors of international patent application PCT/US2013/036763 “Modulation of Angiotensin II Receptors for the Prevention and Treatment of Cerebral Malaria."

Submitted: February 25, 2016; Accepted: July 12, 2016.

Reference information: / Clin Invest. 2016;126(10):4016-4029. doi:10.1172/JCI87306.
Here, we show that rupture of $P f$-iRBCs over human brain microvascular endothelial cells (HBMECs) induces the activation of $\beta$-catenin, which mediates the disruption of IEJs in these cells. Inhibition of $\beta$-catenin activation with modulators of angiotensin II (Ang II) receptors protects endothelial cells from $P f$-iRBC-induced disruption. Some of these modulators, which are approved drugs for use in humans, also induce significant protection of mice from cerebral malaria. We propose that repurposing of these drugs could be used as adjunctive host-targeted therapy for the treatment of cerebral malaria.

\section{Results}

Pf-iRBCs induce the disruption of IEJs in HBMECs. To study the effect of $P f$-iRBCs on brain endothelial cells, we first generated an immortalized cell line from HBMECs that expresses characteristic endothelial markers and is responsive to tumor necrosis factor (TNF) (Supplemental Figure 1; supplemental material available online with this article; doi:10.1172/JCI87306DS1), a key player in the pathophysiology of cerebral malaria (15). To model the interactions of $P f$-iRBCs and brain endothelial cells, HBMEC monolayers were incubated with $P f$-iRBCs (3D7) in the schizont stage for 18 hours (rupture of schizonts is $>90 \%$ under these conditions). The maximal ratio of $P f$-iRBCs to HBMECs used was 40:1, which represents the ratio necessary to cover the surface of the well at 
A

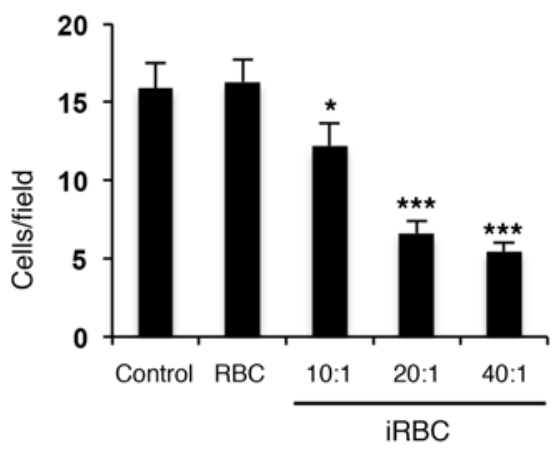

C

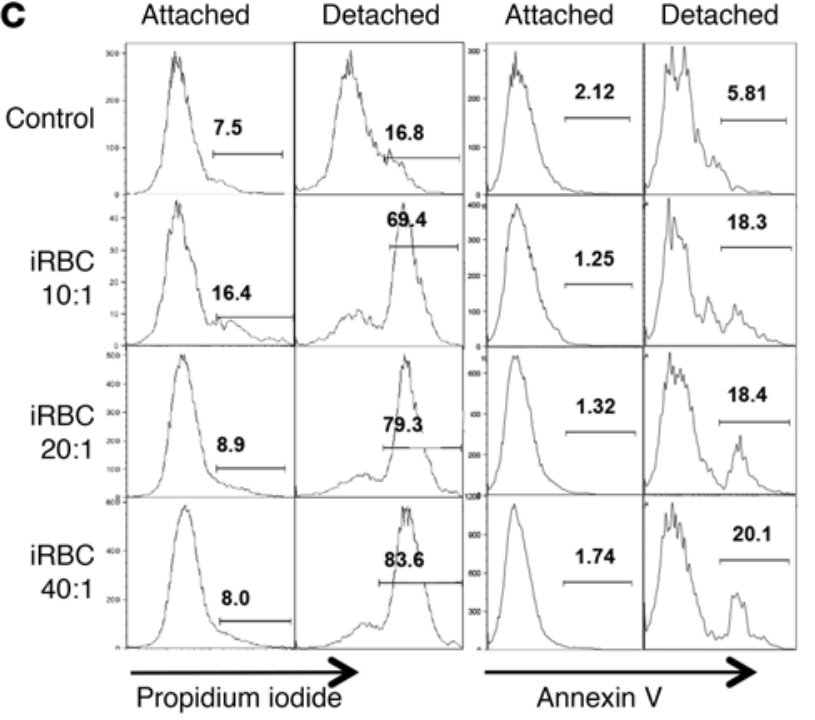

B

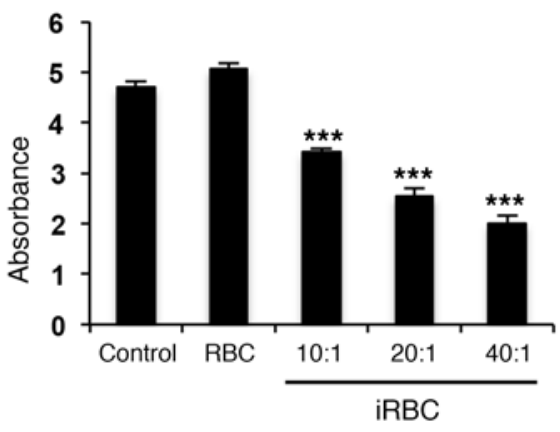

D

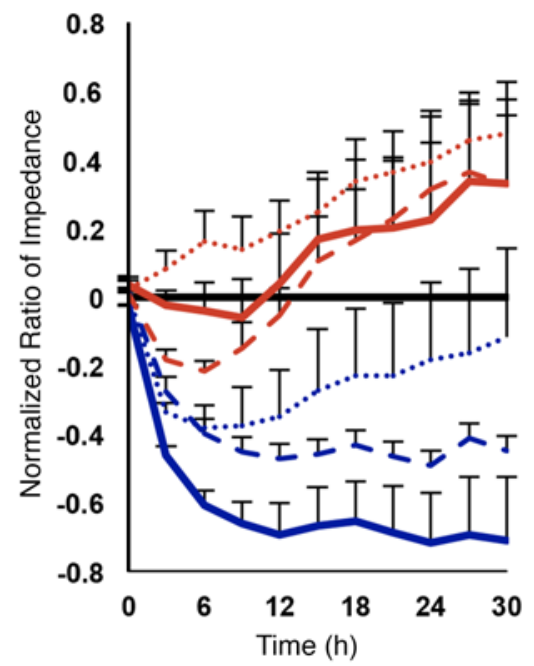

Figure 1. Pf-iRBCs induce a dose-dependent detachment from substrate and disruption of IEJs in HBMECs. Monolayers of HBMECs were incubated with $P f$-iRBCs at the schizont stage or control uninfected RBCs for 18 hours at the indicated ratios of iRBCs to HBMECs or 40:1 for RBCs. (A) Average number of HBMECs per microscopic field $(n=10)$. (B) PrestoBlue determination of viable attached HBMECs. (C) FACS analysis of attached and detached HBMECs stained with propidium iodide or annexin V. (D) Transendothelial electrical resistance (TEER) for HBMEC monolayers incubated with Pf-iRBCs (blue) or control uninfected RBCs (red) at iRBC/HBMEC ratios of 10:1 (dotted line), 20:1 (dashed line), or 40:1 (continuous line), normalized by TEER of HBMECs alone. Results are the average of 6 independent measurements. Results are representative of at least 3 independent experiments. Error bars represent \pm SEM. ${ }^{*} P<0.05,{ }^{* *} P<0.001$ compared with control (1-way ANOVA and Tukey post hoc analysis).

single-layer density, mimicking the pathological accretions of $P f$-iRBCs observed in vivo in brain capillaries. We observed that $P f$-iRBCs induce detachment from the substrate of a fraction of HBMECs, which are found to be apoptotic or dead in the culture media, while virtually all of the remaining attached HBMECs stay alive (Figure 1, A-C). A dose-dependent decrease in transendothelial electrical resistance of the HBMEC monolayers was observed after incubation with $P f$-iRBC schizonts, indicating disruption of the endothelial monolayer integrity (Figure 1D). Staining of IEJ proteins showed an extensive disruption of these structures resulting in the opening of intercellular gaps, and HBMEC detachment from the substrate was observed in a dose-dependent manner (Figure 2A). HBMECs showed a marked decrease in expression of vinculin and ZO-1, which are present in focal adhesions and tight junctions, respectively. We also observed higher levels of expression of $\beta$-catenin, which migrates from adherens junctions to the perinuclear and nuclear areas where it accumulates (Figure $2, \mathrm{~A}-\mathrm{D}) . \beta$-Catenin has a dual role in cells, as structural protein in IEJs in resting conditions, but also as transcriptional activator in the nucleus when cells are activated (16). Migration of $\beta$-catenin into the HBMEC nucleus could therefore be indicative of endothelial activation, a process that has been related to the pathology of cerebral malaria (17).

We also studied the effect of $P$. falciparum on endothelial cells derived from organs other than the brain. We incubated lung and coronary endothelial cell lines with $P f$-iRBC schizonts and found no significant effects on cellular detachment or translocation of $\beta$-catenin (Supplemental Figure 2).

Rupture of $P$. falciparum schizonts is required to induce the HBMEC monolayer disruption and migration of $\beta$-catenin. To determine whether schizont rupture and release of contents is necessary to induce HBMEC monolayer disruption, we incubated schizonts from a $P$. falciparum line that is conditionally deficient in PfCDPK5, a kinase required for parasite egress (18). We observed that arrested schizonts (16.2\% rupture) did not induce disruption of the HBMEC monolayer (Figure $3 \mathrm{~A}$ ) or migration of $\beta$-catenin to the nucleus (Figure $3 \mathrm{~B}$ ) compared with control schizonts (99.4\% rupture). These indicate that rupture, presumably 
A

Control

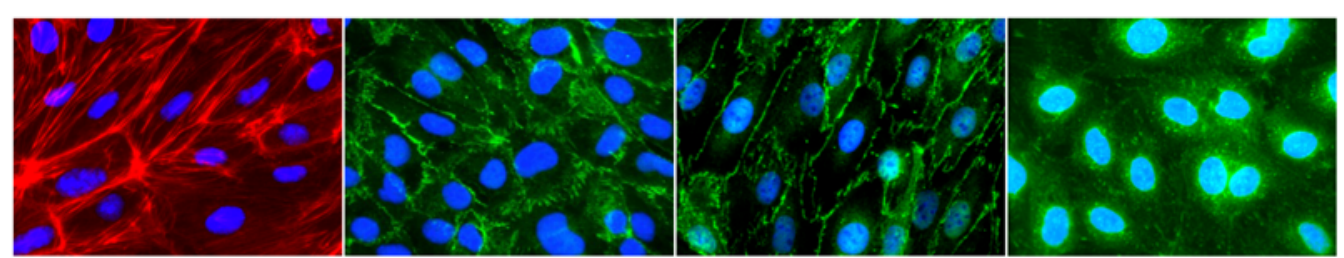

$\mathrm{RBC}$

$40: 1$

$\mathrm{RBC}$

10:1

RBC

20:1
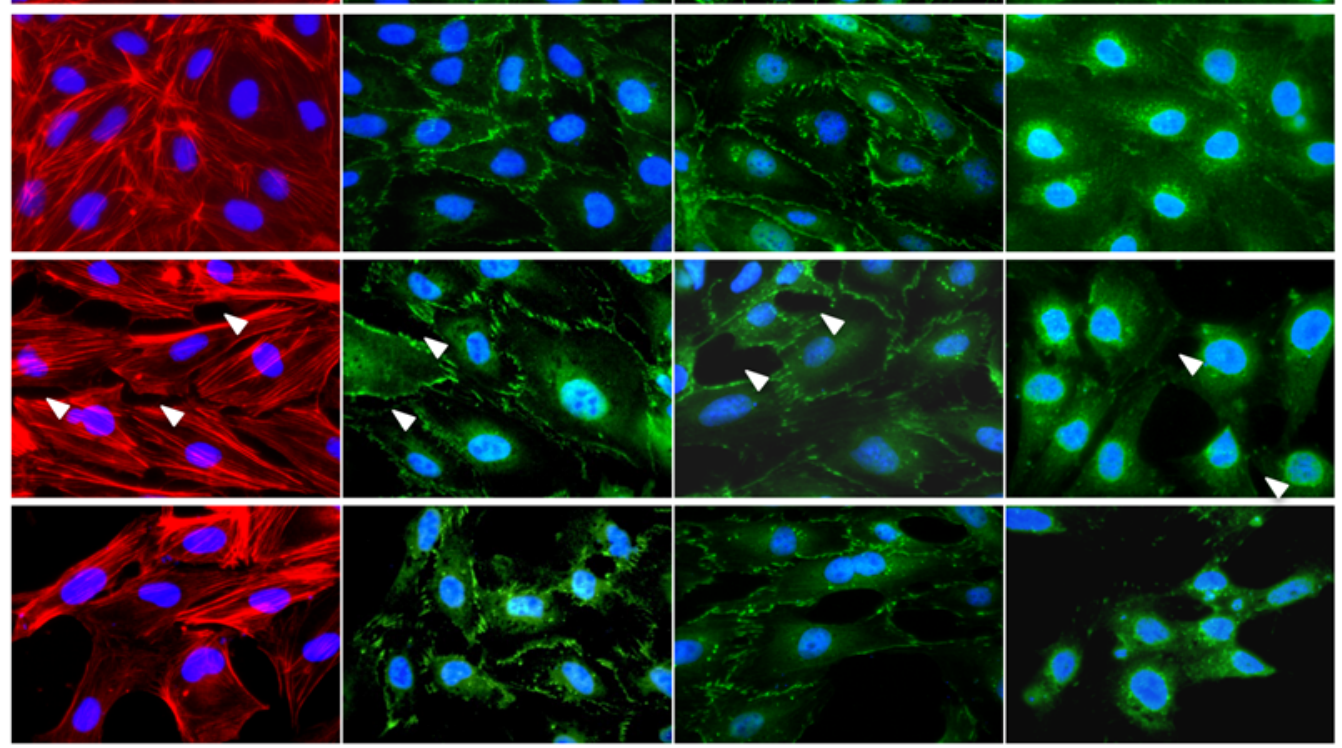

iRBC

40:1

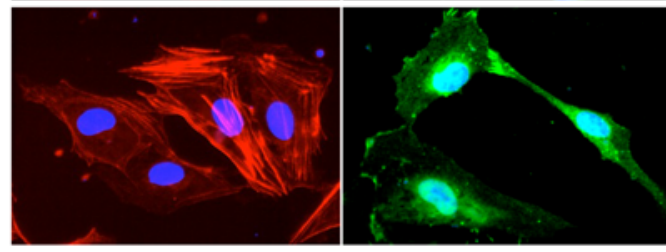

B
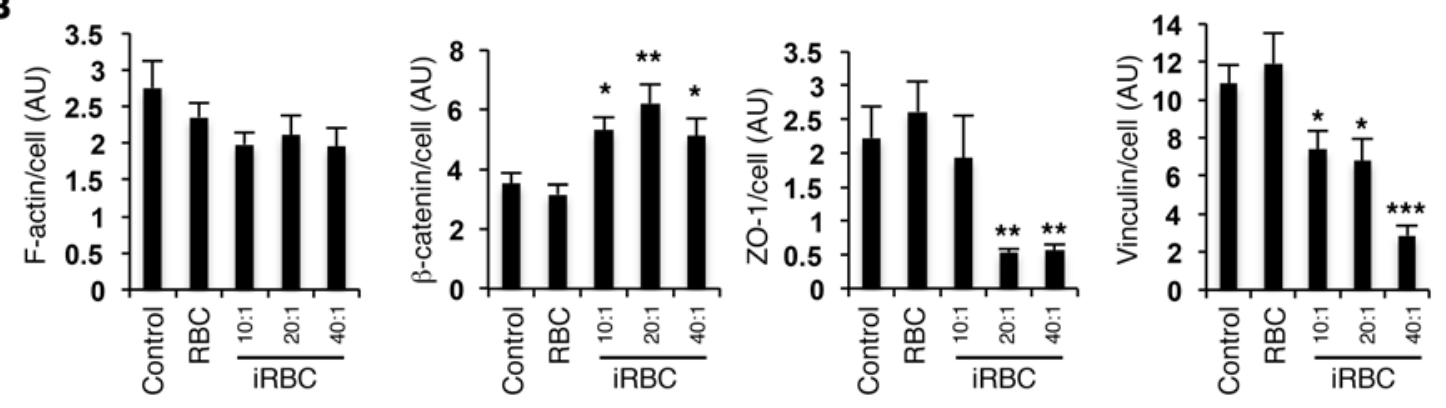

C
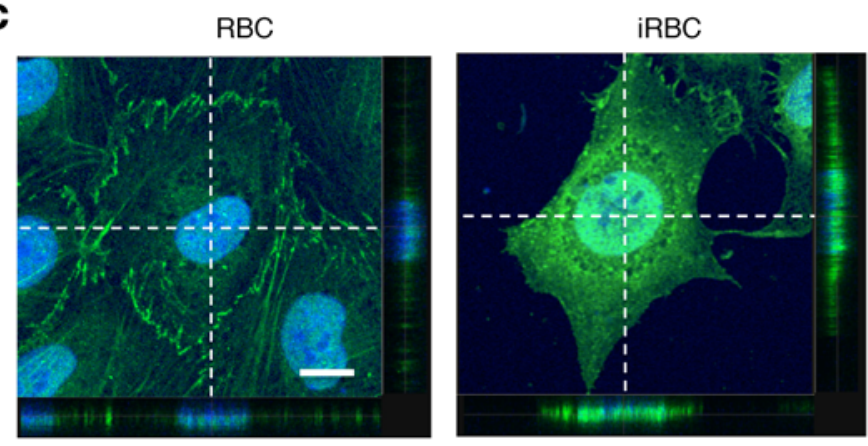

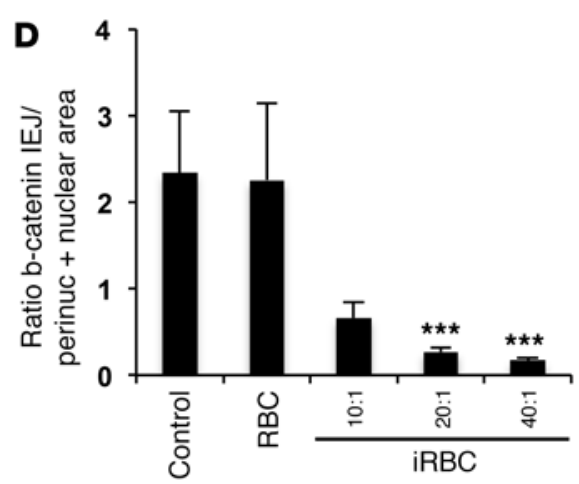


Figure 2. Pf-iRBCs induce disruption of IEJs and migration of $\beta$-catenin to the nucleus. Monolayers of HBMECs were incubated with Pf-iRBCs at the schizont stage or control uninfected RBCs for 18 hours at the indicated ratios of iRBCs to HBMECs or 40:1 for RBCs. (A) HBMEC immunostaining for the indicated proteins (red or green) and nuclei (blue). Examples of opening of intercellular gaps are indicated with arrowheads. Scale bar: $30 \mu \mathrm{m}$. (B) Quantification of fluorescence intensity in arbitrary units (AU) for each protein. (C) Confocal analysis of representative HBMECs incubated with control RBCs or iRBCs and stained for $\beta$-catenin (green) and nuclei (blue). Side views show confocal projections of cells on the dashed-line axes. Colocalization of nuclear DAPI staining and $\beta$-catenin is observed in HBMECs incubated with $P f$-iRBCs. Scale bar: $10 \mu \mathrm{m}$. (D) Quantification of $\beta$-catenin fluoresce intensity in the perinuclear and nuclear areas versus IEJs for each cell in 10 microscopic fields. Results are representative of at least 3 independent experiments. Error bars represent \pm SEM. ${ }^{*} P<0.05$, ${ }^{* *} P<0.01,{ }^{* *} P<0.001$ compared with control. One-way ANOVA and Tukey post hoc analysis for $F$-actin, $\beta$-catenin, and vinculin in B. Kruskal-Wallis test and pairwise comparisons using Dunn's procedure with a Bonferroni correction for multiple comparisons for ZO-1 in B and D.

because of the release of merozoites and/or intraerythrocytic contents, is required to induce the profound disruption of IEJs that we observe in our assay.

Since cytoadhesion of $P f$-iRBCs has been proposed to induce signaling in endothelial cells through the ePCR receptor, leading to IEJ disruption (4), we used the ePCR-cytoadherent P. falciparum line (IT4var19; ref. 2) to determine whether this process mediates the observed disruption of the HBMEC monolayers. We compared the effects of isolated schizonts with knobs (adherent) and without knobs (not adherent) from this line (Supplemental Figure 3) and found similar effects on HBMECs (Figure 3, C and D). These results indicate that rupture, but not cytoadhesion, of $P$. falciparum schizonts is required to induce the observed HBMEC monolayer disruption. Signaling through the ePCR receptor and probably others could still interfere with the permeability of the endothelium and the integrity of IEJs through a pathway different from $\beta$-catenin. In the context of cerebral malaria, both cytoadhesion, which leads to sequestration of schizonts, and the rupture that follows, which would trigger endothelial dysfunction, would be required to induce pathology.

Endothelial cells sense the frictional force of blood flow, which activates intracellular signaling and results in the regulation of gene expression and cellular behavior (19). After subjecting HBMECs to shear stress levels described in the cerebral microvasculature $(\zeta=1$ dyn $\left./ \mathrm{cm}^{2}\right)(20)$ before and during the incubation with nonadherent $P f$-iRBCs, we observed similar results of cellular detachment and translocation of $\beta$-catenin to the nucleus in HBMECs (Figure 3, $\mathrm{E}-\mathrm{G})$. These results confirm that flow conditions do not alter the effect on HBMECs, but also suggest that a $P f$-iRBC-derived soluble factor or factors are responsible for this effect.

To further characterize the nature of the $P f$-iRBC-derived factor(s) triggering the disruption of HBMEC monolayers, we collected the culture medium of $P f$-iRBC schizonts after 18 hours of incubation to allow rupture ( $>90 \%)$. After filtration to eliminate intact merozoites and aggregated materials from ruptured schizonts, the culture medium still preserved the activity to disrupt the endothelial monolayer and translocate $\beta$-catenin (Figure $3, \mathrm{H}$ and I).

$P f-i R B C-$ induced disruption of IEJs is mediated by the activation of $\beta$-catenin. $\beta$-Catenin translocation into the nucleus results in binding to the TCF/LEF transcription factor complex and trans- activation of numerous genes, some of which decrease IEJ stability, causing disruption in different cell types (16). To determine whether $P f$-iRBCs induce the activation of TCF/LEF transcription, we transfected HBMECs with a lentiviral vector that carries the $T c f$ promoter linked to a fluorescent reporter for activation (TCF-eGFP) (21). Incubation of $P f$-iRBCs with HBMECs results in strong activation of the Tcf promoter in transfected cells (Figure 4, $\mathrm{A}$ and $\mathrm{B})$, confirming $P f$-iRBC-dependent activation of $\beta$-catenin in HBMECs. As positive control for $\beta$-catenin activation we used CHIR-99021, a blocker of the main $\beta$-catenin inhibitory regulator (glycogen synthase kinase $3 \beta)(16,22)$, which induced a lower level of activation of $\beta$-catenin compared with $P f$-iRBCs.

We also investigated whether the activation of $\beta$-catenin mediates the observed detachment from the substrate and disruption of IEJs in HBMECs. Using a dominant-negative TCF4 (dnTCF4) lentiviral vector to inhibit $\beta$-catenin-induced transcription (21) (Supplemental Figure 4; see complete unedited blots in the supplemental material.), we observed that inhibition of TCF/ LEF transcription protects HBMEC monolayers from $P f$-iRBCinduced damage (Figure 4, C-E). These results confirm that $P f$-iR$\mathrm{BC}$-induced $\beta$-catenin activation mediates the detachment from the substrate and disruption of IEJs in HBMECs and suggest that its inhibition could be explored for therapeutic purposes.

Modulators of Ang II receptors inhibit $\beta$-catenin activation and disruption of IEJs. To identify inhibitors of $\beta$-catenin activation that could interfere with P. falciparum-induced effects on HBMECs and potentially have a protective effect on the BBB and on the outcome of cerebral malaria, we examined the effects of the 2 receptors of the vasoactive peptide Ang II, since this pathway regulates vascular permeability (23) and has also been associated with decreased susceptibility to cerebral malaria in human malaria patients (24). We first confirmed that Ang II type 1 and type 2 receptors (AT1 and AT2) are expressed in HBMECs (Figure 5A), before testing whether receptor modulators interfere with the activation of $\beta$-catenin induced by $P$. falciparum. Since AT1 and AT2 have counteractive effects in regulating vascular homeostasis and permeability through the regulation of IEJs, we chose a receptor blocker of AT1 (losartan) and an agonist of AT2 (CGP-42112A), which are both expected to decrease vascular permeability (25). We observed that both losartan and CGP-42112A inhibit the activation of $\beta$-catenin induced by $P f$-iRBCs (Figure 5, B and C) and also prevent HBMEC detachment and disruption of IEJs induced by $P f$-iRBCs (Figure 5 , $\mathrm{D}-\mathrm{H})$. These results indicate that modulation of Ang II receptors interferes with the activation of $\beta$-catenin and consequently regulates the stability of the endothelial monolayer.

Modulators of Ang II receptors inhibit cerebral malaria in mice. We have used a mouse model for cerebral malaria (P. berghei ANKA infection of C57BL/6J mice) to study the effects of Ang II receptor modulators on the permeability of the BBB and susceptibility to experimental cerebral malaria during infection. This model does not reproduce the mechanism of initiation of human cerebral malaria, since $P$. berghe $i$ iRBCs are not cytoadhesive; however, after aggregation of blood cells in brain capillaries obstructs circulation, the inflammatory environment, endothelial disruption, and BBB damage induced are similar to those in human cerebral malaria $(26,27)$, providing an adequate model for our studies, which are focused on the protection of the host brain endothelium. 
A

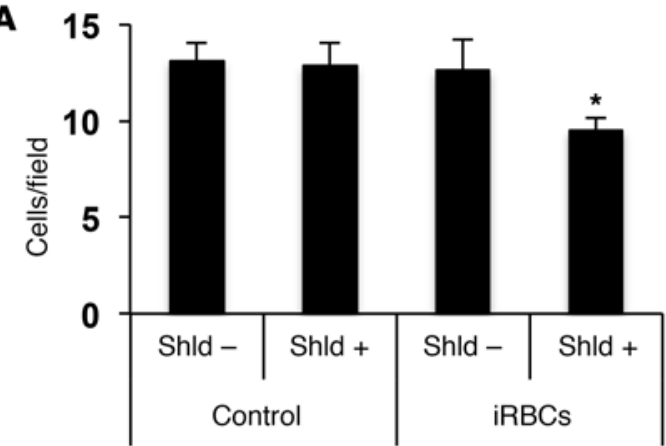

C

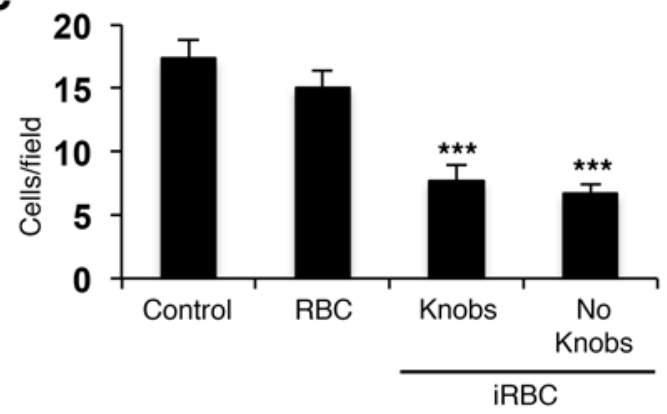

E

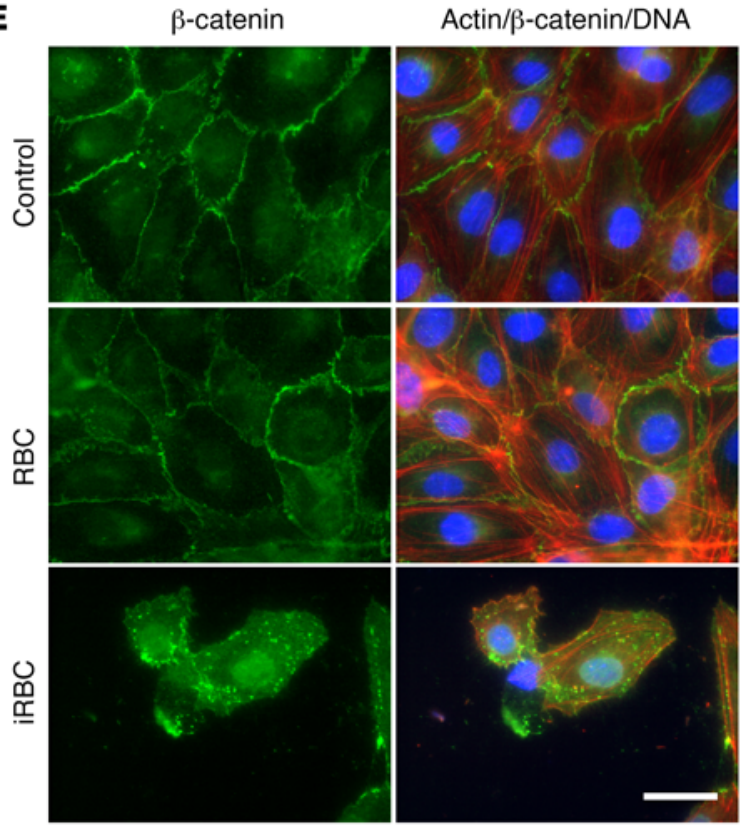

H

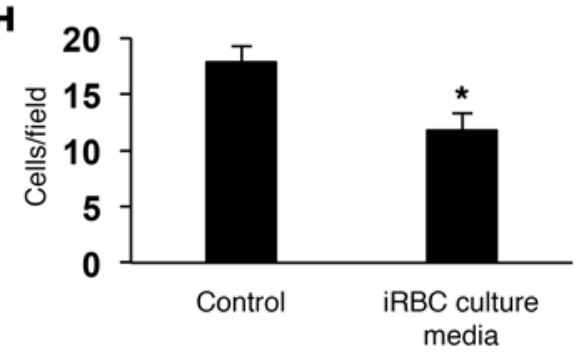

B

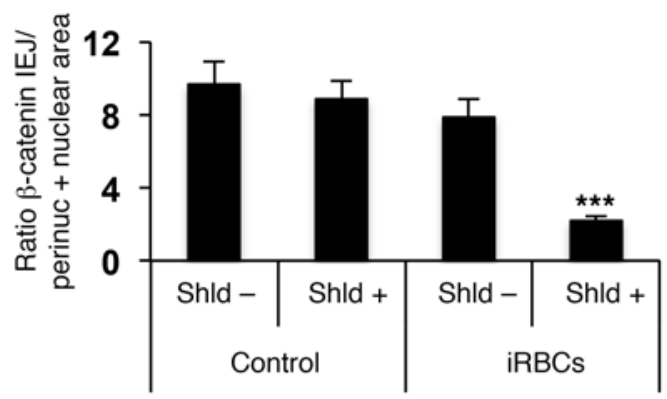

D

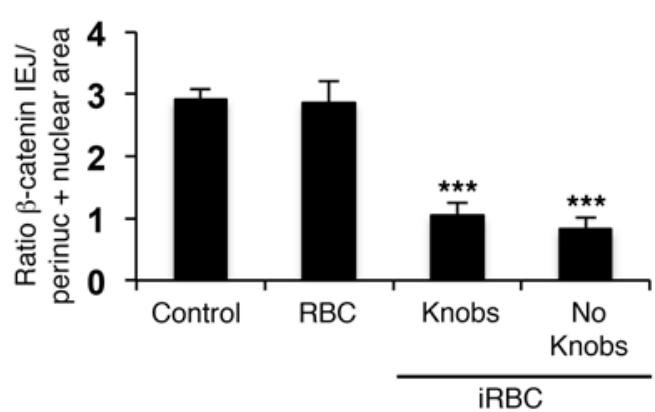

$\mathbf{F}$

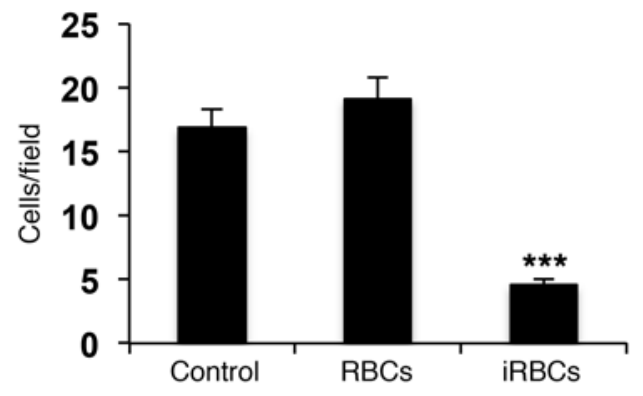

G

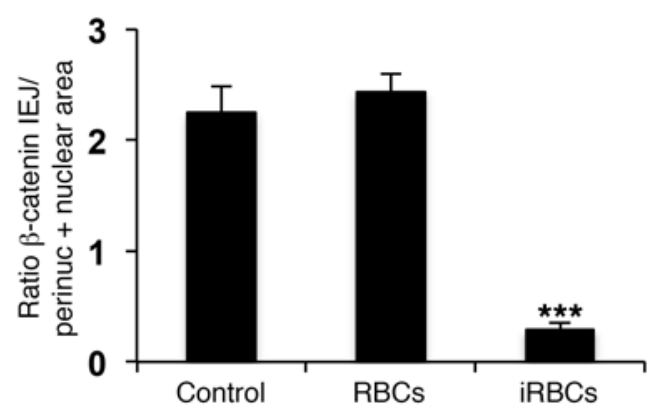

I

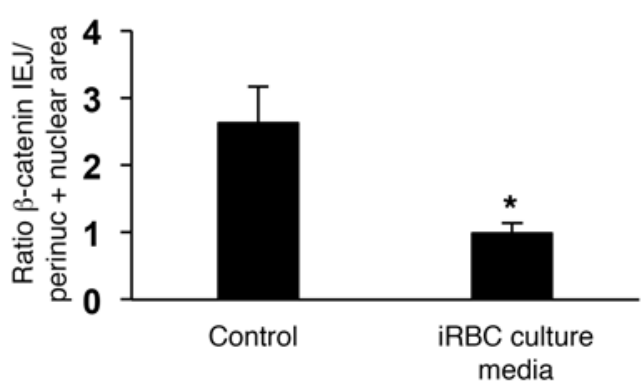


Figure 3. Rupture of $P$. falciparum schizonts, but not cytoadhesion, is required to induce the HBMEC monolayer disruption and migration of $\boldsymbol{\beta}$-catenin. (A and $\mathbf{B}$ ) Monolayers of HBMECs were incubated without parasites (Control) or with D10-PfCDPK5-DD ${ }_{\mathrm{TM}} P$. falciparum schizonts $(1: 40)$ in the absence (-) or presence $(+)$ of Shield-1 (Shld). The presence of Shield-1 allows conditionally CDPK5-deficient arrested schizonts to rupture (18). (C and D) HBMECs were incubated alone, with uninfected RBCs, or with IT4var19 $P$. falciparum schizonts (1:40) with or without knobs. (E-G) HBMECs were incubated alone, with uninfected RBCs, or with $3 D 7 P$. falciparum schizonts (1:40) under flow conditions $\left(\zeta=1 \mathrm{dyn} / \mathrm{cm}^{2}\right)$. HBMEC immunostaining for $\beta$-catenin (green), actin (red), and nuclei (blue) is shown in panel $\mathbf{E}$. Scale bar: $30 \mu \mathrm{m}$. (H and I) HBMECs were incubated alone or with the culture media of ruptured iRBCs. Results are expressed as average number of HBMECs per microscopic field $(n=10)$ in $\mathbf{A}, \mathbf{C}, \mathbf{F}$, and $\mathbf{H}$, and as the quantification of $\beta$-catenin fluorescence intensity in the perinuclear and nuclear areas versus IEJs for each cell in 10 microscopic fields in B, D, G, and $\mathbf{I}$. Results are representative of at least 3 independent experiments. Error bars represent \pm SEM. ${ }^{*} P<0.05,{ }^{* * *} P<0.001$ compared with control. Parametric 2-tailed Student's $t$ test ( $\mathbf{H}$ and $\mathbf{I}$ ) or 1-way ANOVA and Tukey post hoc analysis were applied (A-D, F, and $\mathbf{G})$.

Mice were infected with $P$. berghei ANKA and closely monitored for neurological signs. We observed that treatment with losartan or CGP-42112A since the first day of infection reduces significantly the incidence of experimental cerebral malaria from $75 \%$ in the control group to $13.3 \%$ and $28.5 \%$ in the treated groups, respectively. A genetic approach using AT2-deficient mice (Agtr2 ${ }^{-/-}$mice) (28) showed that absence of this receptor increased susceptibility to experimental cerebral malaria with an earlier onset than in controls and $100 \%$ incidence (Figure 6A). Parasitemia in all groups was similar, indicating that the protective effect against experimental cerebral malaria is not caused by an inhibition of Plasmodium infection (Figure 6B). A previous report showed that mice treated with Ang II are more resistant to cerebral malaria, but they also present lower levels of parasitemia (29) because of the direct toxicity of Ang II to Plasmodium (30). This double effect precludes drawing direct conclusions about the mechanistic effect of Ang II in experimental cerebral malaria (29).

To confirm that the infected mice had developed experimental cerebral malaria, as indicated by the neurological signs, brain histological sections of each mouse were analyzed for the presence of hemorrhages that are characteristic of this syndrome. We found that every infected mouse, even when protected from experimental cerebral malaria, had developed at least a few cerebral hemorrhages, confirming the disruption of the BBB (Figure 6 , C-E). However, mice treated with the 2 Ang II receptor modulators presented lower numbers or smaller size of hemorrhages, suggesting that the damage to the $\mathrm{BBB}$ was mitigated in these groups of mice (Figure 6, D and E). Interestingly, AT2-deficient mice presented lower numbers of hemorrhages, but of larger size than in the control group. Likely, the larger hemorrhages not only triggered the early onset of experimental cerebral malaria but also prevented further increase in the numbers of hemorrhages because of earlier death.

Ang II receptor modulators as adjunctive therapy for cerebral malaria in mice. With the aim of investigating the therapeutic potential of Ang II receptor modulators for cerebral malaria, we modified the mouse model to better represent the conditions of patients in the clinic. Only a small percentage of $P$. falciparum infections develop cerebral malaria, but once neurological signs have appeared, a high proportion of cases (about 20\%) will result in death and $10 \%$ to $20 \%$ of survivors will develop long-term neurological sequelae, even if correct antimalarial treatment is administered (1). Therefore, treatment for cerebral malaria would have to be administered after neurological signs are evident, since there are currently no early clinical predictors for the development of cerebral malaria. Treatment would be administered in combination with conventional anti-Plasmodium medication to eliminate the parasite.

For this analysis, we selected an AT1 blocker that is highly effective and commonly used for the treatment of hypertension (irbesartan; ref. 31) and the only AT2 agonist that is currently in clinical development (compound 21; ref. 32). Treatments were administered only after the appearance of neurological signs in the mice (day 5.5 after infection) and in combination with chloroquine. We observed a high increase in survival rates when irbesartan or compound 21 was added, in comparison with control mice treated only with chloroquine, in which survival is low (Table 1 and Figure 7). It is remarkable to note that a high proportion of mice presenting critical neurological signs at the time when adjunctive treatment was started fully recovered from cerebral malaria. These results establish the basis for repurposing of Ang II modulators as adjunctive therapy for cerebral malaria.

\section{Discussion}

Cerebral malaria is one of the major causes of death in malaria patients; however, the underlying cellular and molecular mechanisms causing this pathology remain widely unknown. Our findings that (a) rupture of $P f$-iRBCs induces the activation of $\beta$-catenin, which leads to the disruption of IEJs in brain endothelial cells, and (b) this process is inhibited by Ang II receptor modulators establish $\beta$-catenin as a key effector of $P$. falciparum in endothelial pathology and open immediate therapeutic possibilities.

Cerebral malaria is characterized by the binding of $P f$-iRBCs to ePCR, ICAM-1, and possibly other molecules, on brain endothelial cells (2-4). This binding recruits high numbers of $P f$ iRBCs to the brain endothelium, creating a microenvironment where circulation is impaired and where release of merozoites and erythrocyte components would accumulate as a result of natural rupture of $P f$-iRBCs at the end of their cycle. Our results indicate that rupture of $P f$-iRBCs triggers the activation of $\beta$-catenin in endothelial cells, but it is likely that binding to ePCR and ICAM- 1 may also activate $\beta$-catenin-independent signaling pathways that decrease the integrity of IEJs, as previously proposed (4), ultimately causing the loss of BBB integrity (Figure 8). Inflammatory cytokines that increase endothelial permeability, such as TNF, could also contribute to this process by destabilizing IEJs in endothelial cells (33). A combination of these factors could lead to vasogenic edema in the brain of cerebral malaria patients, potentially leading to brain swelling and death (34). It is important to note that regardless of the mechanism inducing the disruption of endothelial junctions, the inhibition of $\beta$-catenin activation by Ang II modulators is expected to promote IEJ stability and increase brain endothelial integrity (35). 
A
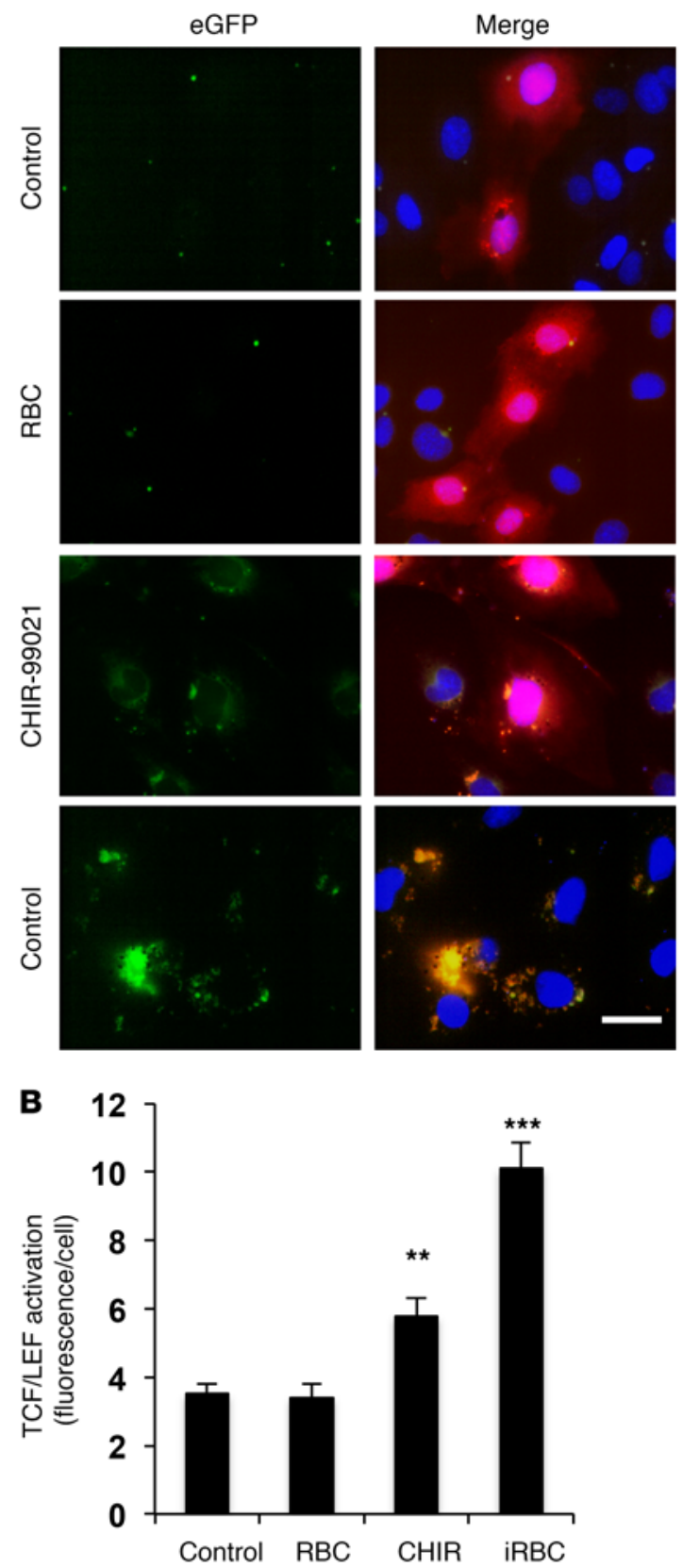

C
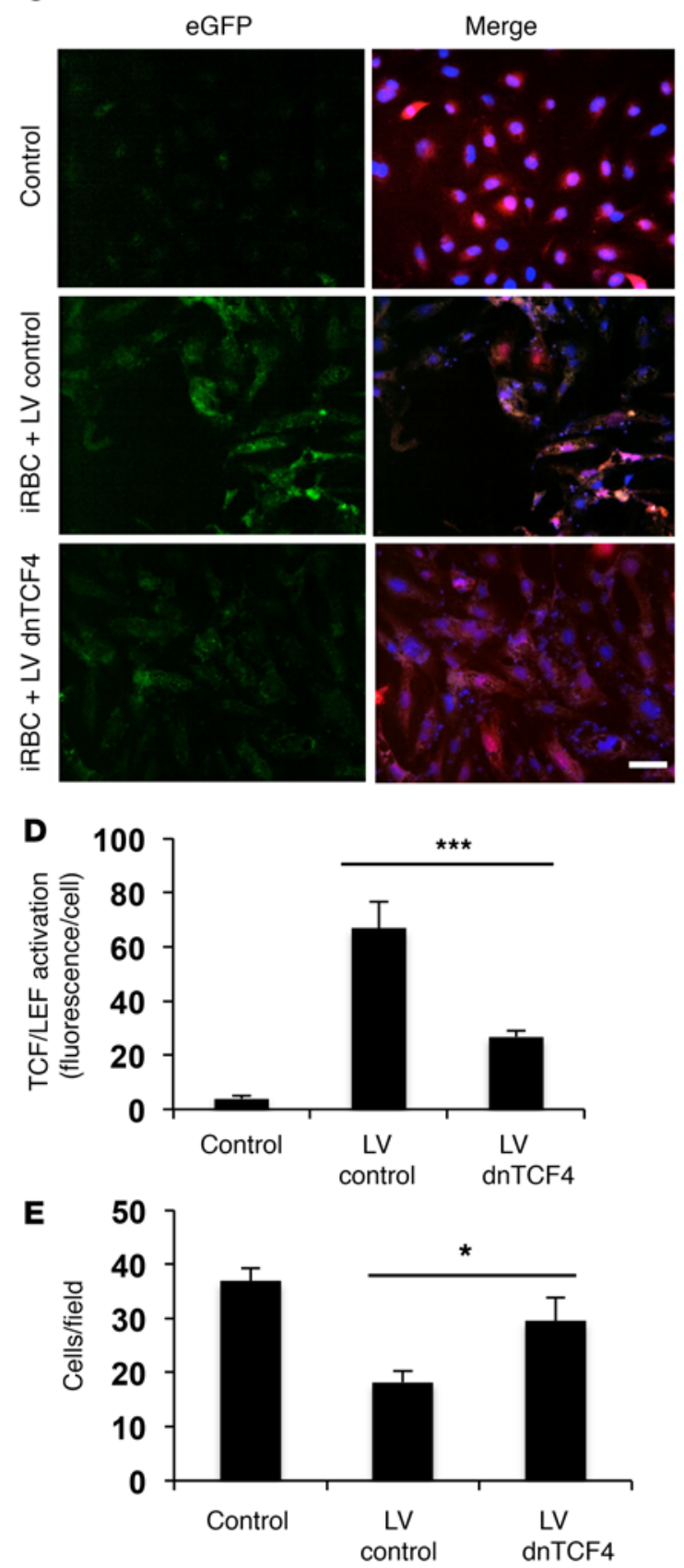

Figure 4. Pf-iRBC-induced disruption of IEJs is mediated by the activation of $\boldsymbol{\beta}$-catenin in HBMECs. HBMEC monolayers were incubated with Pf-iRBCs at the schizont stage or control uninfected RBCs for 18 hours at 40:1 iRBC/HBMEC ratio. (A and B) HBMEC monolayers were infected with lentivirus (constitutively expressing mCherry, red) carrying the TCF-eGFP reporter (eGFP, green when activated). Forty-eight hours later, cells were incubated with RBCs, iRBCs, or CHIR-99021 (a positive control for activation of $\beta$-catenin; ref. 22). (A) The same microscopic field is shown for eGFP alone and merged with mCherry and nuclei. Scale bar: $40 \mu \mathrm{m}$. (B) Quantification of eGFP fluorescence per cell. (C-E) Monolayers of HBMECs expressing constitutively the TCF-eGFP $\beta$-catenin reporter and the mCherry marker were infected or not (Control) with lentivirus carrying dnTCF4 that inhibits $\beta$-catenin-induced transcription (LV dnTCF4) or an empty lentivirus (LV control). Forty-eight hours later, cells were incubated with Pf-iRBCs. (C) The same microscopic field is shown for eGFP alone and merged with mCherry and nuclei. Scale bar: $50 \mu \mathrm{m}$. (D and E) Quantification of eGFP fluorescence per cell (D) or number of cells per microscopic field (E). Results are representative of at least 3 independent experiments. Error bars represent \pm SEM. ${ }^{*} P<0.05,{ }^{*} P<0.01,{ }^{* *} P<0.001$ compared with control. One-way ANOVA for the medians in B and D. One-way ANOVA and Tukey post hoc analysis for E.

Since $\beta$-catenin is a major regulator of cellular adhesion (36), the connection of this pathway with Ang II receptors links together 2 major regulatory pathways in endothelial cells, which has important consequences for the understanding of endothelial physiology and opens therapeutic possibilities for the regulation of brain endothelial integrity and permeability. Our observations from the in vitro experiments together with the genetic and pharmacological approaches in vivo indicate that susceptibility 
to experimental cerebral malaria is regulated by Ang II receptors: protection can be achieved by activation of AT2 (with CGP42112A) or by blockade of AT1 (with losartan), while the opposite effect is observed by deletion of AT2.

Taken together, our results indicate that a shift toward stimulation of the AT2 receptor pathway, which is also achieved through the blocking of AT1, inhibits the activation of $\beta$-catenin, increases the integrity of IEJs, and determines the outcome of experimental cerebral malaria.

Another signaling pathway directly implicated in the integrity of brain endothelium is the angiopoietin/Tie2 axis, which plays a critical role in endothelial activation and is associated with the pathogenesis of cerebral malaria (37). Angiopoietin-1 via its receptor Tie 2 expressed by endothelial cells stabilizes the endothelium by promoting endothelial tight junctions, but these effects are partially inhibited by angiopoietin-2, resulting in endothelial activation and increased vascular permeability (38). Several studies have correlated the levels of angiopoietin-1 and -2 with cerebral malaria severity and survival (37, 39-41). Interestingly, a relation between AT1/AT2 activity and angiopoietin-2 levels has been described in endothelial cells $(42,43)$, possibly mediated by VEGF $(44,45)$, that may link the activities of both pathways in vivo.

Clinicians agree on the need for host-targeted adjunct therapy to rescue patients from death and/or cognitive impairments caused by cerebral malaria (46). Strengthening of the IEJs with modulators of Ang II receptors could be used as a new strategy to develop adjunctive treatments for cerebral malaria that "buy time" while conventional anti-Plasmodium drugs eliminate the parasite. Therefore, we propose a host-targeted therapeutic strategy based on the reinforcement of brain endothelial cell junctions through the inhibition of $\beta$-catenin activation. Repurposing of AT1 blockers, which are commonly used for treatment of hypertension, as adjunctive treatment for cerebral malaria appears as an immediate therapeutic possibility. Blood pressure levels of patients would have to be monitored closely during treatment, as is done routinely when severe malaria infections are treated with quinidine, which also may induce hypotension (47). After its clinical development is completed, compound 21 may also become a suitable alternative for treatment, since AT2 agonists would provide the protective effects mediated by the inhibition of $\beta$-catenin with lower risk of inducing hypotension (48).

The finding that Ang II receptor signaling inhibits the activation of $\beta$-catenin connects 2 major regulatory pathways in brain endothelial cells and suggests that modulators of AT1 and AT2 may have therapeutic potential for diseases in which the disruption of endothelial permeability contributes to pathology, as recently documented for Ebola infections (49).

\section{Methods}

\section{P. falciparum culture}

Erythrocytic asexual cultures of P. falciparum 3D7 and IT4var19, a gift from J. Smith (Center for Infectious Disease Research, Seattle, Washington, USA) (2), were maintained at $5 \%$ hematocrit in complete medium (RPMI 1640, $25 \mathrm{mM}$ HEPES, $10 \mu \mathrm{g} / \mathrm{ml}$ gentamycin, $0.5 \mathrm{mM}$ hypoxanthine, $\mathrm{pH}$ 6.75), supplemented with $25 \mathrm{mM}$ sodium bicarbon- ate and $0.5 \%$ Albumax II for 3D7 or 10\% pooled human serum from 10 different donors (Interstate Blood Bank Inc.) for IT4var19 at 5\% oxygen, $5 \%$ carbon dioxide, and $90 \%$ nitrogen. Parasite cultures were synchronized using magnetic separation of late stages with a MACS cell separation column (Miltenyi Biotec). To monitor the PfEMP1 expression in the IT4var19 late-phase parasites, rabbit anti-IT4var19 DBLa2 serum and a control rabbit serum, both provided by J. Smith (Center for Infectious Disease Research, Seattle) (2), followed by secondary anti-rabbit-Alexa 488 (111-546-047; Jackson ImmunoResearch) were used to stain iRBCs that were analyzed by FACs.

D10-PfCDPK5-DDTM, a gift from J.D. Dvorin (Harvard University, Boston, Massachusetts, USA), are transgenic P. falciparum parasites that have a destabilizing domain (DD) inserted to regulate the expression levels of PfCDPK5, a kinase that controls parasite egress from erythrocytes (18). In the absence of the ligand Shield-1, DD-fused CDPK5 is rapidly degraded and parasites are arrested in the schizont stage. The D10-PfCDPK5-DDTM line was grown in normal media in the presence of $250 \mathrm{nM}$ Shield-1 (Clontech). One day before the experiment, when the culture is synchronized in ring stage, medium was changed to the same medium without Shield-1. After 24 hours, when parasites in culture were arrested in schizont stage, they were separated using a magnetic MACS cell separation column. Schizonts were then divided in 2 and incubated overnight with a confluent monolayer of HBMECs with or without Shield-1. After incubation, medium was recovered and centrifuged at $1,200 \mathrm{~g}$ for $5 \mathrm{~min}$ utes, and the pellet was used to make a smear and stained with Giemsa to calculate the percentage of ruptured iRBCs. Effects on the HBMEC monolayers were quantified by immunofluorescence of $\beta$-catenin and determination of number of cells per field.

To produce the culture medium of ruptured $P f$-iRBCs, late-stage schizonts were resuspended in medium $0.5 \% \mathrm{FBS}$ at $14 \times 10^{6} / \mathrm{ml}$ and cultured for 18 hours to allow for natural rupture and parasite egress. The percentage of rupture was quantified by counting in a hemocytometer. The culture medium was then passed through a $0.22-\mu \mathrm{m}$ nylon filter to remove cellular debris. ECM medium 0.5\% FBS was added to reach a final concentration of ruptured iRBCs equivalent to 8 $\times 10^{6} / \mathrm{ml}$. HBMEC monolayers were then incubated for 18 hours with ECM medium $0.5 \%$ FBS alone or containing the filtered soluble material from $8 \times 10^{6}$ ruptured iRBCs $/ \mathrm{ml}$.

Immortalization and culture of HBMECs and other endothelial cell lines Purified HBMECs were purchased frozen from ScienCell Research Laboratories in passage 1, thawed, seeded on culture flasks, and grown to confluence in DMEM/F12 medium ( $\mathrm{pH}$ 7.4) supplemented with $10 \% \mathrm{FBS}, 30 \mathrm{mg} / \mathrm{ml}$ endothelial cell growth supplement, and $10 \mathrm{mg} / \mathrm{ml}$ penicillin/streptomycin. Cells were incubated at $37^{\circ} \mathrm{C}$ in $5 \% \mathrm{CO}_{2}$ in water-saturated air. Primary HBMECs in their second passage from isolation were infected with Lenti-SV40-Ta virus immortalization kit (Capital Biosciences). Briefly, cells were infected overnight in a 6-well plate with $2 \mathrm{ml} /$ well supernatant in the presence of 8 $\mu \mathrm{g} / \mathrm{ml}$ polybrene. After 18 hours, the viral supernatant was discarded and fresh culture medium was added to the cells before a 72-hour step incubation at $37^{\circ} \mathrm{C}$. Cells were then trypsinized and subcultured in two 12-well plates for 7 days. Monolayers were then observed for growth and morphology changes before being analyzed for the expression of SV40 large T antigen by ELISA. The HBEC-4A clone was selected for our analyses and was characterized for PECAM-1 (303101; BioLegend), VE-cadherin (348507; BioLegend), and EPCR 
A

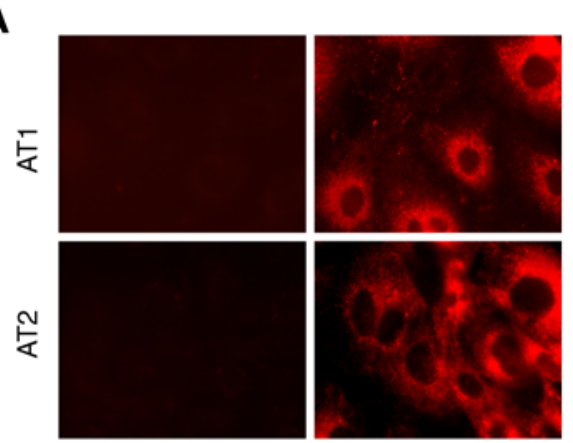

B

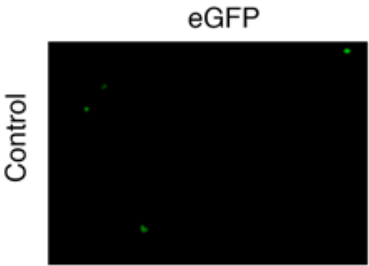

Merge
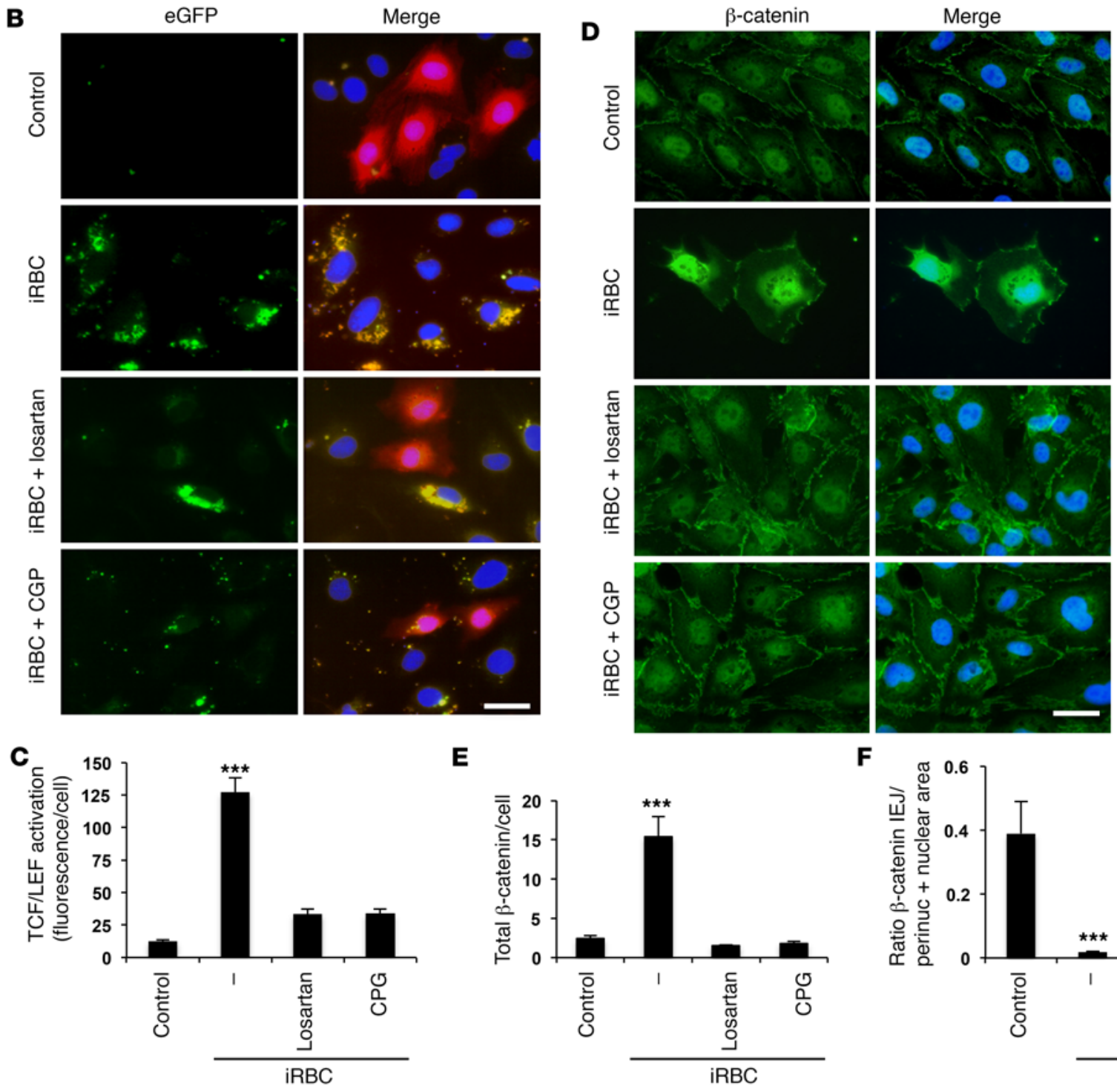

E

G
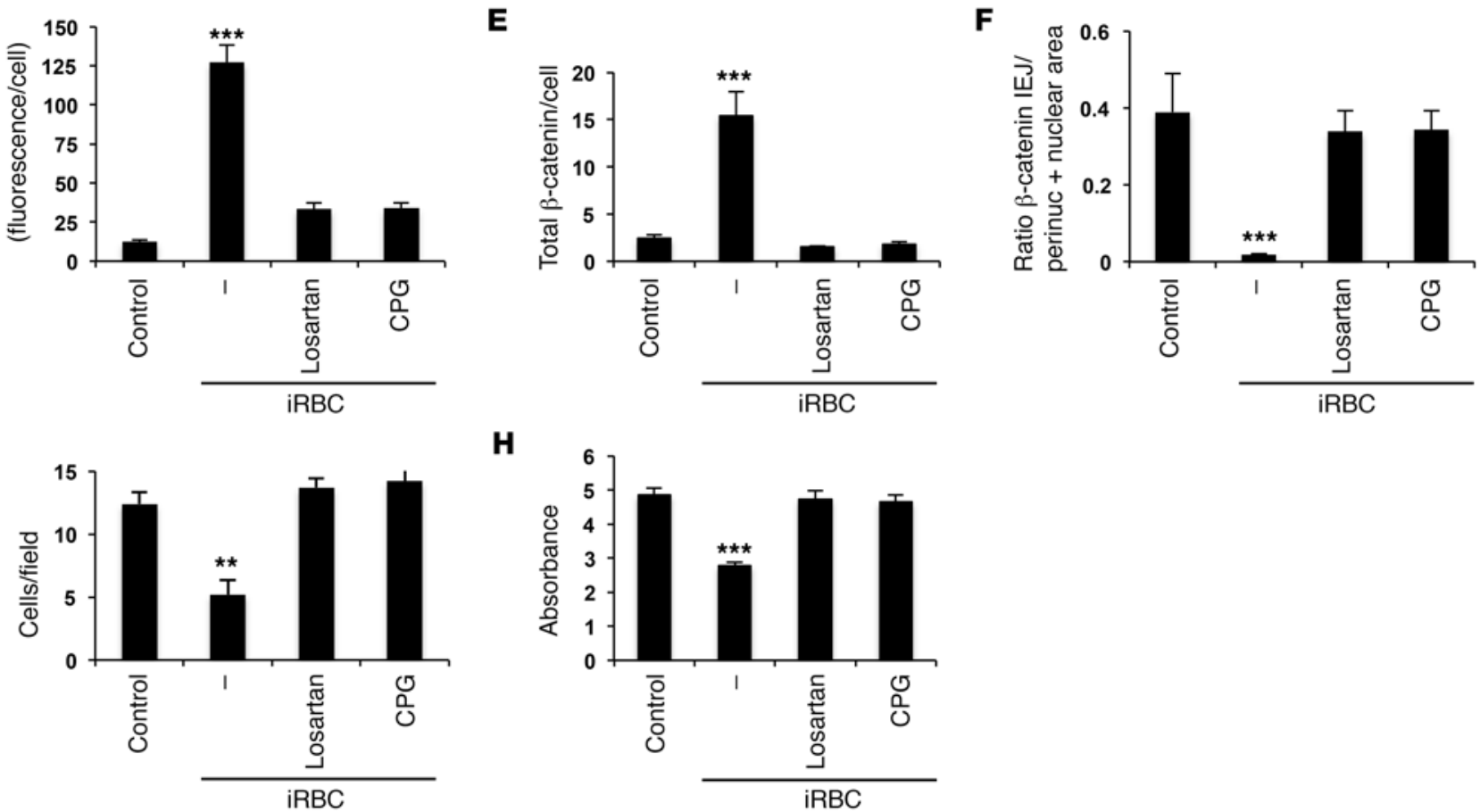

H

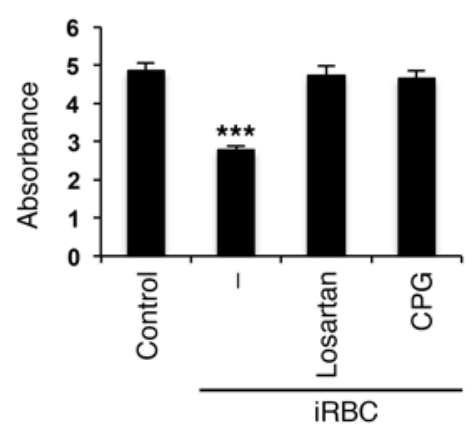


Figure 5. Modulators of Ang II receptors inhibit Pf-iRBC-induced $\beta$-catenin activation, detachment, and disruption of IEJs in HBMECs. (A) HBMEC monolayers were incubated with specific antibodies against AT1 and AT2 receptors followed by a secondary antibody (right panels) or with only the secondary antibody (left panels). (B and C) As in Figure 4A, HBMEC monolayers were infected with $\beta$-catenin reporter lentivirus before incubation with iRBCs alone $(40: 1)$ or together with losartan $\left(10^{-5} \mathrm{M}\right)$ or CGP-42112A ( $\left.10^{-5} \mathrm{M}\right)$ for 18 hours. (B) The same microscopic field is shown for eGFP alone and merged with mCherry and nuclei. (C) Quantification of eGFP fluorescence per cell. (D-H) HBMEC monolayers were incubated with $P f$-iRBCs, losartan, and CGP-42112A as in B followed (D-G) or not (H) by immunostaining for $\beta$-catenin (green) and nuclei (blue). (E-C) Quantification of green fluorescence intensity per cell $(\mathbf{E})$, green fluorescence intensity in the perinuclear and nuclear areas versus IEJs for each cell (F), and number of cells per microscopic field (G). (H) PrestoBlue determination of viable attached HBMECs. Results are representative of at least 2 independent experiments. Scale bar: $40 \mu \mathrm{m}$. Error bars represent \pm SEM. ${ }^{* *} P<0.01,{ }^{* *} P<0.001$ compared with control. Kruskal-Wallis test and pairwise comparisons using Dunn's procedure with a Bonferroni correction for multiple comparisons.

(351903; BioLegend) by flow cytometry in resting conditions. Also cells were analyzed for their response to TNF (BD Pharmingen) (10 $\mathrm{ng} / \mathrm{ml}$ ) for VCAM (305809; BioLegend) and ICAM-1 (313103; BioLegend) (Supplemental Figure 1). HBMECs were routinely cultured in ECM medium with 5\% FBS, $1 \%$ endothelial cell growth supplement, and $10 \mathrm{mg} / \mathrm{ml}$ penicillin/streptomycin (ScienCell).

Human pulmonary microvascular endothelial cells (HPMECs) (50) and human coronary artery endothelial cells (HCAECs) (Cambrex Bio Sciences) were grown in EBM-2 basal medium (CC-3156) supplemented with EGM-2 MV singleQuot kit that includes 5\% FBS and VEGF (CC-4147; Lonza). HPMECs (provided by C.J. Kirkpatrick, Johannes Gutenberg University, Mainz, Germany) and HCAECs (provided by E. Fisher, New York University, New York, New York, USA) were seeded on gelatin-coated glass coverslips at $5.5 \times 10^{4}$ and $8 \times 10^{4}$ cells $/ \mathrm{ml}$ density, respectively, 24 hours before incubation with $P f$-iRBCs was started.

\section{Flow conditions}

HBMECs $\left(160,000\right.$ cells) were seeded onto a $\mu$-Slide I Luer ${ }^{0.2}$ (Ibidi $\mathrm{GmbH}$, Germany) and incubated at $37^{\circ} \mathrm{C}$ and $5 \% \mathrm{CO}_{2}$ for $30 \mathrm{~min}-$ utes for cell attachment. Reservoirs in the chamber were then filled with supplemented ECM media, and cells were incubated an additional 2 hours to allow them to form a confluent monolayer. Cells were then subjected for 4 hours to flow conditions using a peristaltic minipump (Thermo Fisher Scientific). The flow was adjusted to produce shear stress levels described in the cerebral microvasculature $\left(\zeta=1 \mathrm{dyn} / \mathrm{cm}^{2}\right)$ (20). After that time, $P f$-iRBCs or uninfected RBCs were added to the flow system at the same concentration that is used for ratios of 40:1 iRBCs/HBMECs in static-conditions experiments $\left(8 \times 10^{6} / \mathrm{ml}\right)$ and allowed to circulate for 18 hours before fixation and processing for immunofluorescence.

\section{Cytoadhesion assay}

IT4var19 parasites were cultured as described above. iRBCs with knobs were isolated using gelatin flotation. Briefly, cultured parasites were centrifuged 5 minutes at 1,200 $\mathrm{g}$. Pellet was recovered and resuspended with 10 volumes of warmed $0.7 \%$ porcine gelatin (G2625; Sigma-Aldrich) solution in RPMI at $37^{\circ} \mathrm{C}$. After 1 hour of incubation, the upper phase containing an enriched fraction of
iRBCs with knobs was recovered, washed with warm medium, and passed through a magnetic MACS cell separation column (Miltenyi Biotec) to increase purity of late phases. In parallel, the pellet containing knob-negative iRBCs was washed with warm medium, and late-phase parasites without knobs were isolated using a MACS cell separation column.

Cytoadhesion assays $(n=5)$ were carried out by coincubation of confluent monolayers of HBMECs with iRBCs with or without knobs (45 minutes, $37^{\circ} \mathrm{C}$ ) on an orbital shaker (ratio 1:40 HBMECs/iRBCs). After incubation, unbound iRBCs were gently washed off 3 times with medium. After fixation of cells with $4 \%$ paraformaldehyde, adherent iRBCs were determined by counting of 5 random fields per assay under $\times 400$ magnification.

\section{Cell viability assay}

To assess the viability of adherent HBMECs we used the PrestoBlue Cell Viability Reagent (Invitrogen). HBMECs were seeded $\left(1 \times 10^{4}\right.$ cells per well) in 96-well plates 24 hours before addition of RBCs or iRBCs and the different stimuli ( $n=6$ per condition). After 18 hours, the plates were washed twice with PBS, and PrestoBlue Cell Viability Reagent diluted 1:10 with medium was added. Samples were then incubated for 3 hours at $37^{\circ} \mathrm{C}$ before measurement of absorbance at $595 \mathrm{~nm}$.

FACS analysis for apoptosis of attached and detached HBMECs was carried out with FITC Annexin V Apoptosis Detection Kit I (556547; BD Biosciences) according to the manufacturer's recommended protocol.

\section{Measurements of transendothelial electrical resistance of HBMECs}

HBMECs were grown to confluence on gold-plated electrodes in 96-well array slides linked to a cell-substrate impedance sensing system (ECIS; Applied Biophysics). Once a stable measurement of resistance from HBMECs was obtained $(\sim 1,800 \Omega)$, different concentrations of iRBCs and control noninfected RBCs were added, and the resistance changes were monitored in real time for up to 32 hours.

\section{Immunofluorescence microscopy}

HBMECs were seeded on gelatin-coated glass coverslips at $5.5 \times 10^{4}$ cells/ml density 24 hours before. Coverslips were fixed with $4 \%$ paraformaldehyde for 10 minutes at room temperature, followed by permeabilization with $0.25 \%$ Triton X-100 for 10 minutes. Cells were preincubated with blocking buffer (PBS, 10\% goat serum, 1\% BSA, $100 \mathrm{mM}$ glycine; $\mathrm{pH}$ 7.0) for 1 hour at room temperature. Primary rabbit antisera ( $\beta$-catenin, 9587 from Cell Signaling; vinculin, 700062, and ZO-1, 339100, from Invitrogen; AT1, AAR-011 from Alomone Labs; and AT2 sc-9040 from Santa Cruz) were diluted in blocking buffer and incubated overnight at $4^{\circ} \mathrm{C}$. Alexa 488-labeled (111-546-047; Jackson ImmunoResearch) or Alexa 594-labeled (A11020; Life Technologies) secondary anti-rabbit antibodies diluted 1:500 in blocking buffer were incubated for 1 hour at room temperature. Rhodaminelabeled phalloidin (Invitrogen) was diluted 1:2,000 and incubated for 10 minutes at room temperature. DAPI (Sigma-Aldrich) at $1 \mu \mathrm{g} / \mathrm{ml}$ was incubated for 5 minutes at room temperature. Images were acquired and analyzed with MetaMorph Advanced version 7.6.5.0 on an Olympus IX70 inverted microscope.

\section{Confocal microscopy}

HBMEC monolayers were imaged with an inverted Leica TCS SP2 AOBS confocal system ( $\times 40$ HCX PL APO 1.25-0.75 oil lens) with the 
A

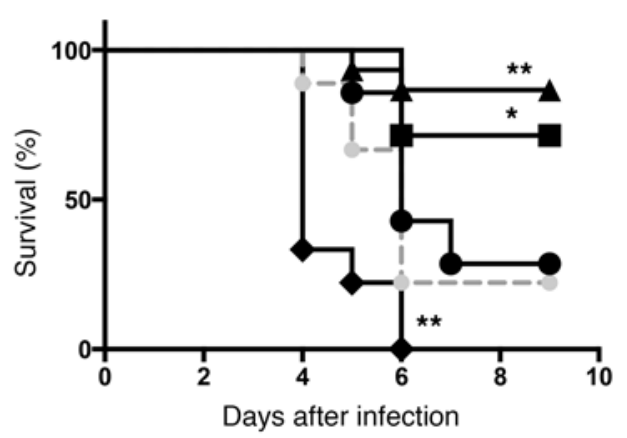

C

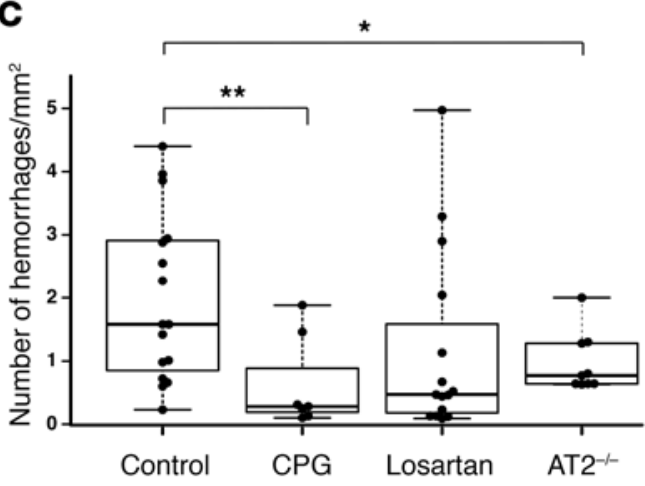

E

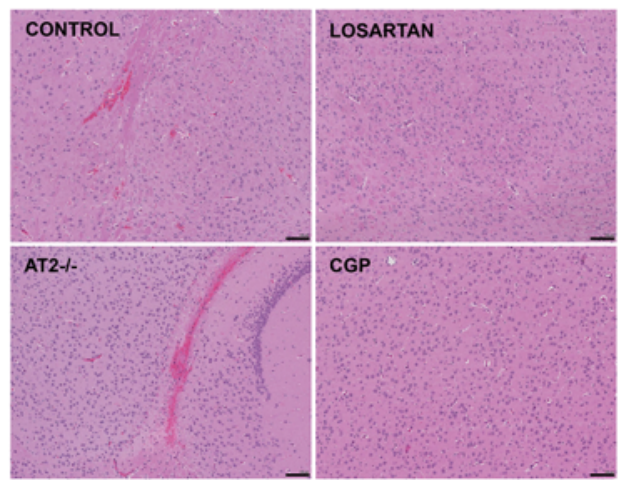

B

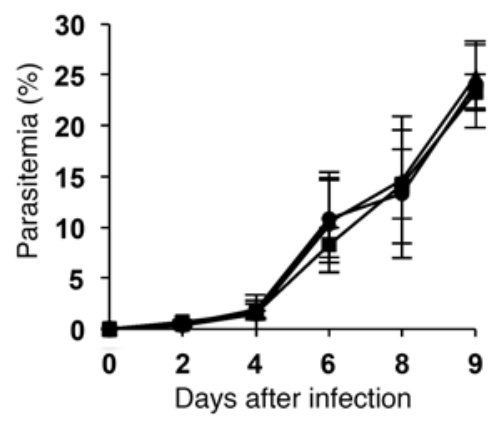

D

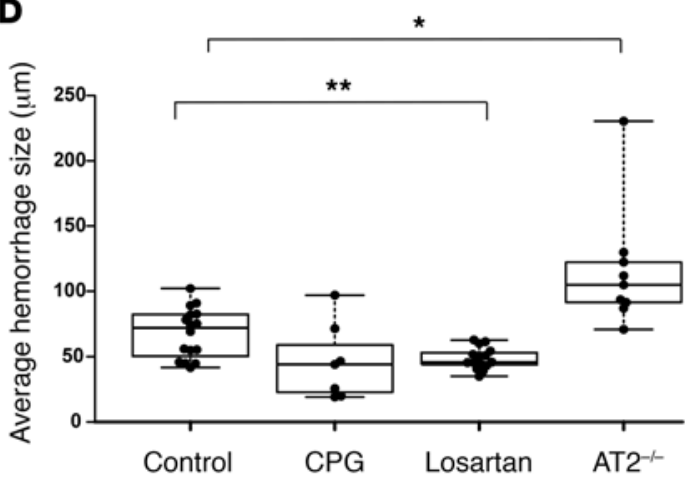

Figure 6. Modulators of Ang II receptors inhibit experimental cerebral malaria. Groups of WT or AT2-deficient (AT2 ${ }^{-/-}$, black diamonds) C57BL/6J mice ( $n=38$ and 9 , respectively) were infected with $P$. berghei ANKA. Starting on the day of infection, WT mice were treated with losartan (in drinking water $10 \mathrm{mg} / \mathrm{kg} / \mathrm{d} ; n=15$; triangles) or CGP-42112A (administered through intradermal micropumps $500 \mathrm{ng} / \mathrm{min} / \mathrm{kg} ; n=7 ;$ squares). Control mice drinking regular water ( $n=7$; black circles) or implanted with intradermal micropumps delivering saline ( $n=9$; gray circles) were used. (A and B) Survival and mean parasitemia. (C-E) Histological sections of the brains of mice were analyzed for the presence (C) and size (D) of hemorrhages. Control group includes mice drinking regular water and implanted with intradermal micropumps delivering saline. Center lines show the medians; box limits indicate the 25th and 75th percentiles as determined by R software; whiskers extend to minimum and maximum values. (E) Representative microscopic fields. Scale bars: $100 \mu \mathrm{m}$. Error bars represent \pm SEM. ${ }^{*} P<0.05,{ }^{* *} P<0.01$ compared with control. Kaplan-Meier and pairwise log rank after Bonferroni correction in $\mathbf{A}$. One-way repeated-measures ANOVA for $\mathbf{B}$. One-way ANOVA for the medians in $\mathbf{C}$ and $\mathbf{D}$.

following excitation light sources: $405 \mathrm{~nm}$ blue diode laser; $488 \mathrm{~nm}$ argon/krypton line; and HeNe laser lines at $543 \mathrm{~nm}, 594 \mathrm{~nm}$, and 633 $\mathrm{nm}$. Bidimensional reconstruction was performed using Leica LCS software. Colocalization of nuclear DAPI staining and $\beta$-catenin was processed in Imaris 7.4 (Bitplan).

\section{Lentivirus production and viral transduction}

Lentiviral vectors used to probe the Wnt signaling pathway have been previously described (21). The 7xTCF-eGFP//SV40-mCherry (7TGC) (where eGFP expression is driven by a Wnt-responsive cassette) and hEF1 $\alpha$-dnTCF4//SV40-Puro ${ }^{\mathrm{R}}$ (a dominant-negative form of TCF4) viral vectors were a gift from Roel Nusse (Addgene plasmid numbers 24304 and 24311, respectively). Lentiviruses were produced in 293FT cells with plasmids VSVG, pLP1, and pLP2 using the ViraPower Lentiviral Packaging Mix from Life Technologies. As a control for the dnTCF 4 virus, a puromycin-resistant lentivirus (pLKO1) was used as a control for mock infection. Expression of the dnTCF 4 was confirmed by Western blotting (details in Supplemental Information). Lentivi- 
Table 1. Adjunctive therapy with irbesartan (AT1 blocker) or compound 21 (AT2 agonist) decreases mortality from cerebral malaria in mice

$\begin{array}{lcccc} & \text { Control } & \text { Chloroquine } & \text { Chloroquine + irbesartan } & \text { Chloroquine + C21 } \\ \text { Severe signs } & 0^{\mathrm{B}} / 10^{\mathrm{C}} & 2 / 8 & 3 / 3 & 4 / 4 \\ \text { Critical signs }^{\mathrm{D}} & 0 / 10 & 2 / 14 & 8 / 14 & 7 / 11 \\ \text { Total survival } & 0 / 20(0 \%)^{\mathrm{E}} & 4 / 22(18 \%) & 11 / 17(65 \%)^{\mathrm{F}} & 11 / 15(73 \%)^{\mathrm{F}}\end{array}$

${ }^{A}$ Mice with a score of $3 .{ }^{B}$ Number of surviving mice (complete clearance of parasitemia). ${ }^{C}$ Total number of mice. ${ }^{D}$ Mice with a score of 4 to 5 . EPercentage of surviving mice. After Bonferroni correction, statistical significance was accepted at $P<0.0167$. ${ }^{\mathrm{F}} P<0.01$ compared with chloroquine-alone group. of 3 were considered severe cases and with 4 to 5 critical cases of cerebral malaria. Mice were treated with chloroquine alone $(20 \mathrm{mg} / \mathrm{kg} / \mathrm{d}$, i.p.; Sigma-Aldrich) or in combination with irbesartan (50 mg/kg/d, oral gavage; Cayman Chemical Co.) or compound $21(0.3 \mathrm{mg} / \mathrm{kg} / \mathrm{d}$, i.p.; provided by Vicore Pharma). Treatments were started on day 5.5 to day 7 after infection, when mice presented severe or critical signs, and continued until parasitemia was completely cleared (days 11-16 after infection) or signs had reached score 5 and included paralysis for more than 5 minutes and panting (mice were euthanized). ral supernatants were concentrated using the Lenti-X Concentrator from Clontech. Viruses were titrated using 293T cells. HBMECs were infected at a multiplicity of infection of 20:1 (lentivirus/HBMECs) in culture medium containing $8 \mu \mathrm{g} / \mathrm{ml}$ polybrene. Medium was changed the next day before addition of RBCs or iRBCs.

\section{Mice}

Female C57BL/6J mice 4 to 6 weeks old were infected by i.p. inoculation of $1 \times 10^{6}$ erythrocytes infected with P. berghei ANKA. Mice were monitored twice daily for cerebral malaria signs.

Experiments with losartan and CGP-42112A. AT2 knockout mice and their age- and sex-matched WT controls were used from the breeding colony of Thomas Walther at the Forschungseinrichtung für Experimentelle Medizin, Berlin, Germany, and obtained as described $(51,52)$. Mice were considered positive for cerebral malaria when they presented at least 2 of the following signs: ruffled coat, coat starring, panting, hemi- or paraplegia, tendency to roll over on stimulation, ataxia, and convulsions. Mice with paralysis, convulsions, and/or coma were euthanized. This occurred from day 5 to day 9 after infection, depending on the individual animals and groups. Mice that did not develop cerebral malaria were euthanized on day 9. Immediately after euthanasia, brains were collected and washed in cold PBS to remove excess of blood and fixed with paraformaldehyde $4 \%$ for up to 24 hours and subsequently transferred to ethanol $70 \%$.

Osmotic minipumps (model 1002; ALZA Scientific Products) were implanted subcutaneously into C57BL/6J mice at 5 weeks of age. Pumps were filled with either saline (vehicle) or CGP-42112A (Sigma-Aldrich) that was delivered at $500 \mathrm{ng} / \mathrm{min} / \mathrm{kg}$ for the duration of the study. Pumps were placed into the subcutaneous space of mice anesthetized with isoflurane (Henry Schein) through a small incision on the animal's back slightly posterior of the shoulder blades that was closed with Reflex $7 \mathrm{~mm}$ wound closure system (ALZA Scientific Products). All incision sites healed rapidly without any further complication. Implantation of osmotic minipumps does not alter susceptibility to cerebral malaria or parasitemia in mice (29). Losartan (Sigma-Aldrich) was administered in the drinking water and adjusted to a final dose of $10 \mathrm{mg} / \mathrm{kg} / \mathrm{d}$.

Experiments with irbesartan and compound 21. Female C57BL/6 mice 4-6 weeks old were purchased from Taconic Laboratories. Determination and severity of experimental cerebral malaria were scored based on appearance (normal $=0$; coat ruffled $=1$; coat starring/panting =2) and neurological involvement (normal = 0; hunched $=1$; partial paralysis $=2$; convulsions $=3$ ). This scoring system is a modification of a method described before (53). Mice with a score

\section{Histopathological studies}

In order to analyze the presence of hemorrhages in the brain, paraffin-embedded transverse sections of brain ( $4 \mu \mathrm{m}$ thick) were stained with H\&E and scanned on the Leica Biosystems SCN4OO whole-slide scanner. The number and maximal diameter of petechiae and hemorrhages were quantified in an area of $27.4 \pm 1.8 \mathrm{~mm}^{2}$ of brain histological sections per mouse using Slidepath Digital Image Hub (DIH) software.

\section{Statistics}

To test whether the analyzed variables followed a normal distribution, all groups were subjected to a normality Kolmogorov-Smirnov test. Homogeneity of variances was analyzed by Levene statistic. Those variables that confirmed a normal distribution as well as the homoscedasticity principle within the different groups were further analyzed by parametric 2-tailed Student's $t$ test or 1-way ANOVA test for comparison of the media. Otherwise, the nonparametric Kruskal-Wallis test was applied. When presence of outliers was noticed using box plot, robust statistical methods were applied to minimize the impact of the outliers in the study $(54,55)$. Number and size of hemorrhages of the experimental cerebral malaria model were analyzed with robust methods using R statistical soft-

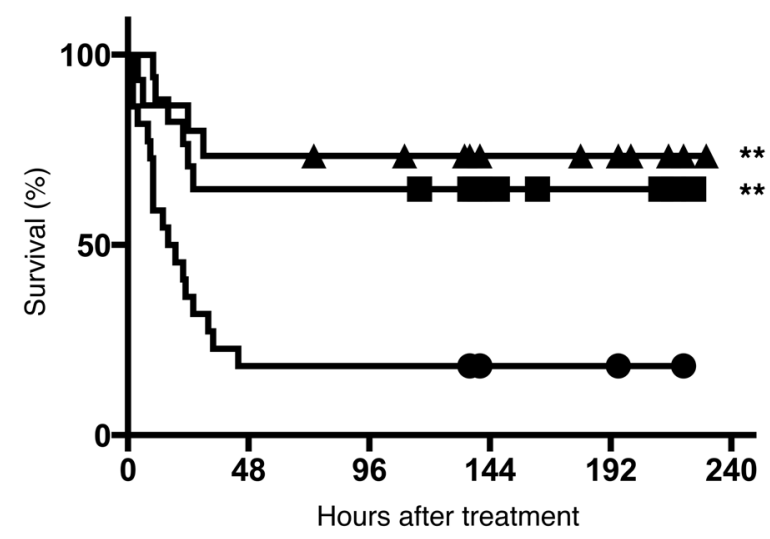

Figure 7. Adjunctive therapy with irbesartan (AT1 blocker) or compound 21 (AT2 agonist) decreases mortality from cerebral malaria in mice. Groups of $[57 \mathrm{BL} / 6]$ mice (see Table 1) were infected with $P$. berghei ANKA and treated with chloroquine alone ( $n=22$; circles), chloroquine plus C21 ( $n=15$; triangles), or chloroquine plus irbesartan ( $n=17$; squares) after they started presenting signs of cerebral malaria (score 3 or higher). ${ }^{* *} P<0.01$ compared with chloroquine-alone group. Kaplan-Meier and pairwise log rank after Bonferroni correction. 

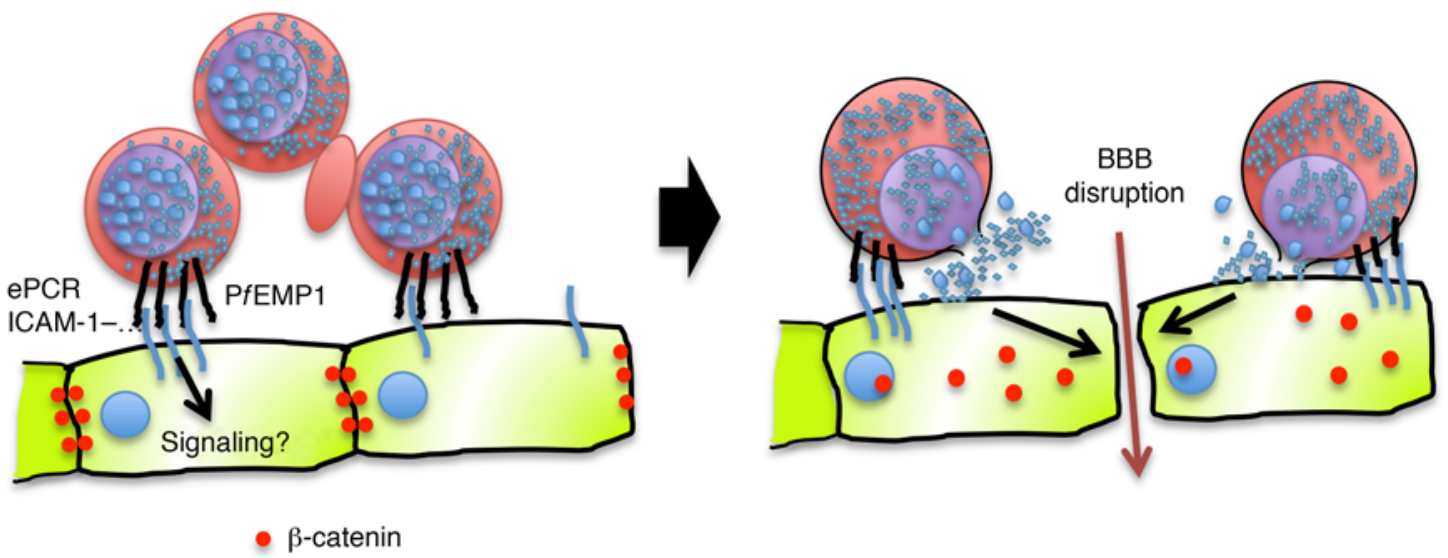

Figure 8. Proposed model for disruption of IEJs during cerebral malaria. First, cytoadhesion of iRBCs to endothelial receptors congregates high densities of iRBCs next to endothelial cells and possibly induces intracellular signaling. After iRBC rupture, release of intraerythrocytic materials triggers the activation of $\beta$-catenin in endothelial cells, which mediates the disruption of IEJs.

ware. One-way ANOVA for the medians based in the generalization of Welch's method and the corresponding post hoc test were performed with the "t1way" and "lincon" functions, respectively, from the package WRS2.

For cerebral malaria determination and survival in mice, log rank test (Kaplan-Meier) was run to determine differences in the appearance of cerebral malaria for the different groups. Pairwise log rank comparisons were conducted after Bonferroni correction was applied to determine which groups had different survival distributions. In vitro statistical analyses as well as parasitemia and log rank test from the in vivo determination of cerebral malaria were carried out using IBM SPSS Statistics version 20 software.

$P$ less than 0.05 was considered significant for all studies except pairwise log rank comparisons, for which $P$ less than 0.0167 was used as reference for signification.

\section{Study approval}

Animal procedures carried out in Spain were performed according to the guidelines of animal research in the European Community, with prior approval by the Ethics Committee of Health Research of the Instituto de Investigación Sanitaria Fundación Jiménez Díaz. All animals were maintained under pathogen-free conditions with an artificial 12-hour dark-light cycle, with free access to food and water. Animal procedures carried out in the United States were performed in strict accordance with the recommendations in the Guide for the Care and Use of Laboratory Animals of the NIH. The protocol was approved by the Institutional Animal Care and Use Committee of New York University School of Medicine, which is fully accredited by the Association for Assessment and Accreditation of Laboratory Animal Care International. All animals were maintained under pathogen-free conditions with an artificial 12-hour dark-light cycle, with free access to food and water.

\section{Author contributions}

JGD designed research studies, conducted experiments, acquired data, analyzed data, and wrote the manuscript. UBR designed research studies and conducted experiments. MT and MA conducted experiments and acquired data. CFA conducted experiments, acquired data, and analyzed data. AM conducted experiments, acquired data, and analyzed data. WX, PG, AW, and IE conducted experiments and acquired data. SW conducted experiments. TW analyzed data and provided reagents. MRO designed research studies, analyzed data, and provided reagents. AR designed research studies, analyzed data, and wrote the manuscript.

\section{Acknowledgments}

Funding was provided by NIH/National Heart, Lung, and Blood Institute (NHLBI) 1R01HL130630 and a Dana Foundation Program in the Neuroimmunology of Brain Infections and Cancers Grant to A. Rodriguez; Fundacion Española para la Ciencia y la Tecnologia, mobility program, Ministry of Education of Spain to J. Gallego-Delgado; NIH Training Grant 5T32AIO07180-30 to M. Ty; Red de Investigación Renal (REDinREN; RD12/0021), Comunidad de Madrid (Fibroteam; S2010/BMD-2321) to M. Ruiz-Ortega; and NIH/National Institute of Allergy and Infectious Diseases U19AI089676-01S1 to S. Wassmer. We thank J. Smith for providing P. falciparum IT4var19 and related reagents, J.D. Dvorin for providing P. falciparum D10-PfCDPK5-DD ${ }_{\mathrm{TM}}$, Vicore Pharma for providing compound 21, C.J. Kirkpatrick for providing HPMECs, and E. Fisher for providing HCAECs.

Address correspondence to: Ana Rodriguez, NYU-Parasitology, 341 East 25th Street, New York, New York 10010, USA. Phone: 212.263.6757; E-mail: Ana.Rodriguez@nyumc.org.
1. Storm J, Craig AG. Pathogenesis of cerebral malaria - inflammation and cytoadherence. Front Cell Infect Microbiol. 2014;4:100.

2. Avril M, et al. A restricted subset of var genes mediates adherence of Plasmodium falciparum-infected erythrocytes to brain endothelial cells. Proc Natl Acad Sci U S A. 2012;109(26):E1782-E1790.

3. Claessens A, et al. A subset of group A-like var genes encodes the malaria parasite ligands for binding to human brain endothelial cells. Proc Natl Acad Sci U S A. 2012;109(26):E1772-E1781.
4. Turner L, et al. Severe malaria is associated with parasite binding to endothelial protein $\mathrm{C}$ receptor. Nature. 2013;498(7455):502-505.

5. Dorovini-Zis K, et al. The neuropathology of fatal cerebral malaria in malawian children. $A m J$ Pathol.2011;178(5):2146-2158. 
6. Brown HC, et al. Blood-brain barrier function in cerebral malaria and CNS infections in Vietnam. Neurology. 2000;55(1):104-111.

7. Gillrie MR, et al. Src-family kinase dependent disruption of endothelial barrier function by Plasmodium falciparum merozoite proteins. Blood. 2007;110(9):3426-3435.

8. Jambou R, Combes V, Jambou MJ, Weksler BB, Couraud PO, Grau GE. Plasmodium falciparum adhesion on human brain microvascular endothelial cells involves transmigration-like cup formation and induces opening of intercellular junctions. PLoS Pathog. 2010;6(7):e1001021.

9. Wilson NO, et al. Soluble factors from Plasmodium falciparum-infected erythrocytes induce apoptosis in human brain vascular endothelial and neuroglia cells. Mol Biochem Parasitol. 2008;162(2):172-176.

10. Tripathi AK, Sha W, Shulaev V, Stins MF, Sullivan DJ. Plasmodium falciparum-infected erythrocytes induce NF- $\mathrm{kB}$ regulated inflammatory pathways in human cerebral endothelium. Blood. 2009;114(19):4243-4252.

11. Tripathi AK, Sullivan DJ, Stins MF. Plasmodium falciparum-infected erythrocytes decrease the integrity of human blood-brain barrier endothelial cell monolayers. Jinfect Dis. 2007;195(7):942-950.

12. Zougbédé S, et al. Metabolic acidosis induced by Plasmodium falciparum intraerythrocytic stages alters blood-brain barrier integrity. JCereb Blood Flow Metab. 2011;31(2):514-526.

13. Steyers CM, Miller FJ. Endothelial dysfunction in chronic inflammatory diseases. Int JMol Sci. 2014;15(7):11324-11349.

14. Brown $\mathrm{H}$, et al. Blood-brain barrier function in cerebral malaria in Malawian children. Am J Trop Med Hyg. 2001;64(3-4):207-213.

15. Hunt NH, Grau GE. Cytokines: accelerators and brakes in the pathogenesis of cerebral malaria. Trends Immunol. 2003;24(9):491-499.

16. Bazzoni G, Dejana E. Endothelial cell-to-cell junctions: molecular organization and role in vascular homeostasis. Physiol Rev. 2004;84(3):869-901.

17. Kim H, Higgins S, Liles WC, Kain KC. Endothelial activation and dysregulation in malaria: a potential target for novel therapeutics. Curr Opin Hematol. 2011;18(3):177-185.

18. Dvorin JD, et al. A plant-like kinase in Plasmodium falciparum regulates parasite egress from erythrocytes. Science. 2010;328(5980):910-912.

19. Baeyens N, Bandyopadhyay C, Coon BG, Yun S, Schwartz MA. Endothelial fluid shear stress sensing in vascular health and disease. J Clin Invest. 2016;126(3):821-828.

20. Adams Y, Rowe JA. The effect of anti-rosetting agents against malaria parasites under physiological flow conditions. PLoS One. 2013;8(9):e73999.

21. Fuerer C, Nusse R. Lentiviral vectors to probe and manipulate the Wnt signaling pathway. PLoS One. 2010;5(2):e9370.

22. Kim H, et al. Modulation of $\beta$-catenin function maintains mouse epiblast stem cell and human embryonic stem cell self-renewal. Nat Commun. 2013;4:2403.
23. Su JB. Vascular endothelial dysfunction and pharmacological treatment. World J Cardiol. 2015;7(11):719-741.

24. Dhangadamajhi G, Mohapatra BN, Kar SK, Ranjit M. Gene polymorphisms in angiotensin I converting enzyme (ACE I/D) and angiotensin II converting enzyme (ACE2 $\mathrm{C} \rightarrow \mathrm{T}$ ) protect against cerebral malaria in Indian adults. Infect Genet Evol. 2010;10(2):337-341.

25. Chung O, Kühl H, Stoll M, Unger T. Physiological and pharmacological implications of AT1 versus AT2 receptors. Kidney Int Suppl. 1998;67:S95-S99.

26. de Souza JB, Hafalla JC, Riley EM, Couper KN. Cerebral malaria: why experimental murine models are required to understand the pathogenesis of disease. Parasitology. 2010;137(5):755-772.

27. Engwerda C, Belnoue E, Grüner AC, Rénia L. Experimental models of cerebral malaria. Curr Top Microbiol Immunol. 2005;297:103-143.

28. Ichiki T, et al. Effects on blood pressure and exploratory behaviour of mice lacking angiotensin II type-2 receptor. Nature. 1995;377(6551):748-750.

29. Gallego-Delgado J, et al. Angiotensin II moderately decreases Plasmodium infection and experimental cerebral malaria in mice. PLoS One. 2015;10(9):e0138191.

30. Saraiva VB, et al. Impairment of the Plasmodium falciparum erythrocytic cycle induced by angiotensin peptides. PLoS One. 2011;6(2):e17174.

31. Bramlage $P$, Schindler C. Differences in pharmacology and their translation into differences in clinical efficacy - a comparison of the renin angiotensin blocking agents irbesartan and losartan. Expert Opin Pharmacother. 2010;11(4):521-535.

32. Steckelings UM, Paulis L, Namsolleck P, Unger T. AT2 receptor agonists: hypertension and beyond. Curr Opin Nephrol Hypertens. 2012;21(2):142-146.

33. Walsh SV, Hopkins AM, Nusrat A. Modulation of tight junction structure and function by cytokines. Adv Drug Deliv Rev. 2000;41(3):303-313.

34. Seydel KB, et al. Brain swelling and death in children with cerebral malaria. $N$ Engl JMed. 2015;372(12):1126-1137.

35. Stamatovic SM, Keep RF, Andjelkovic AV. Brain endothelial cell-cell junctions: how to "open" the blood brain barrier. Curr Neuropharmacol. 2008;6(3):179-192.

36. Valenta T, Hausmann G, Basler K. The many faces and functions of $\beta$-catenin. $E M B O J$. 2012;31(12):2714-2736.

37. Conroy AL, et al. Angiopoietin-2 levels are associated with retinopathy and predict mortality in Malawian children with cerebral malaria: a retrospective case-control study ${ }^{*}$. Crit Care Med. 2012;40(3):952-959.

38. Yuan HT, Khankin EV, Karumanchi SA, Parikh SM. Angiopoietin 2 is a partial agonist/antagonist of Tie2 signaling in the endothelium. Mol Cell Biol. 2009;29(8):2011-2022.

39. Conroy AL, et al. Whole blood angiopoietin-1 and -2 levels discriminate cerebral and severe (non-cerebral) malaria from uncomplicated malaria. Malar J. 2009;8:295.
40. Lovegrove FE, et al. Serum angiopoietin-1 and -2 levels discriminate cerebral malaria from uncomplicated malaria and predict clinical outcome in African children. PLoS One. 2009;4(3):e4912.

41. Yeo TW, et al. Angiopoietin-2 is associated with decreased endothelial nitric oxide and poor clinical outcome in severe falciparum malaria. Proc Natl Acad Sci U S A. 2008;105(44):17097-17102.

42. Yu C, Luo X, Duquette N, Thorin-Trescases N, Thorin E. Knockdown of angiopoietin like-2 protects against angiotensin II-induced cerebral endothelial dysfunction in mice. Am J Physiol Heart Circ Physiol. 2015;308(5):H386-H397.

43. Otani A, Takagi H, Oh H, Koyama S, Honda Y. Angiotensin II induces expression of the Tie2 receptor ligand, angiopoietin-2, in bovine retinal endothelial cells. Diabetes. 2001;50(4):867-875.

44. Benndorf R, Böger RH, Ergün S, Steenpass A, Wieland T. Angiotensin II type 2 receptor inhibits vascular endothelial growth factor-induced migration and in vitro tube formation of human endothelial cells. Circ Res. 2003;93(5):438-447.

45. Hansen TM, Moss AJ, Brindle NP. Vascular endothelial growth factor and angiopoietins in neurovascular regeneration and protection following stroke. Curr Neurovasc Res. 2008;5(4):236-245.

46. Newton CR, Krishna S. Severe falciparum malaria in children: current understanding of pathophysiology and supportive treatment. Pharmacol Ther. 1998;79(1):1-53.

47. Malaria Treatment (United States). Center for Disease Control and Prevention. https://www.cdc. gov/malaria/diagnosis_treatment/treatment.html. Updated June 10, 2015. Accessed July 18, 2016.

48. Danyel LA, Schmerler P, Paulis L, Unger T, Steckelings UM. Impact of AT2-receptor stimulation on vascular biology, kidney function, and blood pressure. Integr Blood Press Control. 2013;6:153-161.

49. Fedson DS, Rordam OM. Treating Ebola patients: a 'bottom up' approach using generic statins and angiotensin receptor blockers. Int J Infect Dis. 2015;36:80-84.

50. Krump-Konvalinkova V, Bittinger F, Unger RE, Peters K, Lehr HA, Kirkpatrick CJ. Generation of human pulmonary microvascular endothelial cell lines. Lab Invest. 2001;81(12):1717-1727.

51. Maul B, et al. Impaired spatial memory and altered dendritic spine morphology in angiotensin II type 2 receptor-deficient mice. J Mol Med. 2008;86(5):563-571.

52. Gembardt F, et al. Cardiovascular phenotype of mice lacking all three subtypes of angiotensin II receptors. FASEB J. 2008;22(8):3068-3077.

53. Waknine-Grinberg JH, et al. Artemisone effective against murine cerebral malaria. Malar J. 2010;9:227.

54. Wilcox R, Carlson M, Azen S, Clark F. Avoid lost discoveries, because of violations of standard assumptions, by using modern robust statistical methods. JClin Epidemiol. 2013;66(3):319-329.

55. Wilcox RR, Keselman HJ. Modern regression methods that can substantially increase power and provide a more accurate understanding of associations. Eur JPers. 2012;26(3):165-174. 